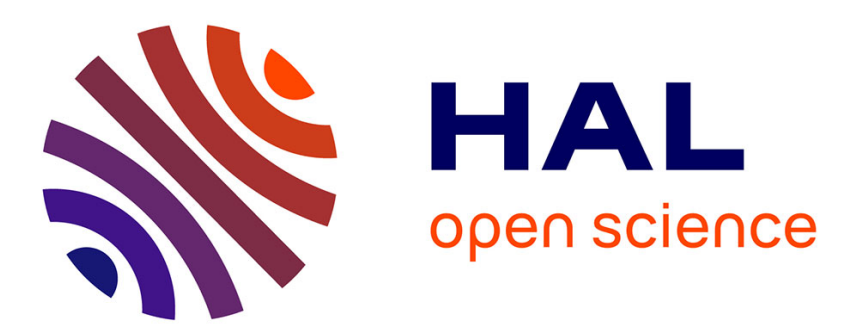

\title{
Computational benefits of structural plasticity, illustrated in songbirds
}

Remya Sankar, Nicolas P. Rougier, Arthur Leblois

\section{To cite this version:}

Remya Sankar, Nicolas P. Rougier, Arthur Leblois. Computational benefits of structural plasticity, illustrated in songbirds. Neuroscience and Biobehavioral Reviews, inPress, 132, pp.1183-1196. 10.1016/j.neubiorev.2021.10.033 . hal-03416314

\section{HAL Id: hal-03416314 \\ https://hal.science/hal-03416314}

Submitted on 5 Nov 2021

HAL is a multi-disciplinary open access archive for the deposit and dissemination of scientific research documents, whether they are published or not. The documents may come from teaching and research institutions in France or abroad, or from public or private research centers.
L'archive ouverte pluridisciplinaire HAL, est destinée au dépôt et à la diffusion de documents scientifiques de niveau recherche, publiés ou non, émanant des établissements d'enseignement et de recherche français ou étrangers, des laboratoires publics ou privés. 


\title{
Computational benefits of structural plasticity, illustrated in songbirds
}

\author{
Remya Sankar ${ }^{1,2,3, \dagger}$ and Nicolas P.Rougier ${ }^{1,2,3,4, \dagger}$ and Arthur Leblois ${ }^{2,3, \dagger}$ \\ ${ }^{1}$ Inria Bordeaux Sud-Ouest, Talence, France; \\ ${ }^{2}$ Institut des Maladies Neurodégénératives, Université de Bordeaux, Bordeaux, France; \\ ${ }^{3}$ Institut des Maladies Neurodégénératives, CNRS, UMR 5293; \\ ${ }^{4}$ LaBRI, Université de Bordeaux, INP, CNRS, UMR 5800, Talence, France; \\ ${ }^{\dagger}$ These authors have contributed equally to this work. \\ *Corresponding author: Arthur.Leblois@u-bordeaux.fr
}

\begin{abstract}
The plasticity of nervous systems allows animals to quickly adapt to a changing environment. In particular, the structural plasticity of brain networks is often critical to the development of the central nervous system and the acquisition of complex behaviors. As an example, structural plasticity is central to the development of song-related brain circuits and may be critical for song acquisition in juvenile songbirds. Here, we review current evidences for structural plasticity and their significance from a computational point of view. We start by reviewing evidence for structural plasticity across species and categorizing them along the spatial axes as well as the along the time course during development. We introduce the vocal learning circuitry in zebra finches, as a useful example of structural plasticity, and use this specific case to explore the possible contributions of structural plasticity to computational models. Finally, we discuss current modelling studies incorporating structural plasticity and unexplored questions which are raised by such models.
\end{abstract}




\section{Contents}

1 Introduction $\quad 3$

2 Structural plasticity 4

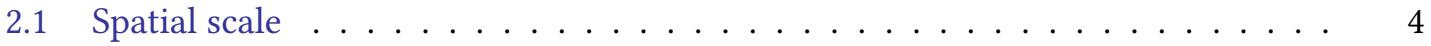

2.2 Time course across development . . . . . . . . . . . . . . 5

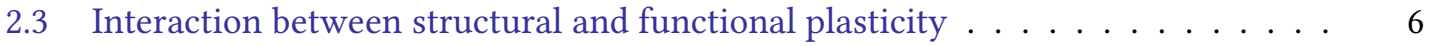

3 From structure to neural activity to song $\quad 7$

3.1 General behavior and brain anatomy in birds . . . . . . . . . . . . 7

3.2 Avian vocal learning . . . . . . . . . . . . . . . . . 9

3.3 Structural plasticity in songbirds . . . . . . . . . . . . . . . . . 13

3.4 Functional impact of structural plasticity . . . . . . . . . . . . . 15

4 From biology to machine learning 16

5 Conclusion $\quad 20$ 


\section{Introduction}

Plasticity is defined as the capacity of the neural activity generated by an experience to modify neural circuit function and thereby modify subsequent thoughts, feelings, and behavior according to [1]. This definition of plasticity encompasses a broad range of phenomena that have been observed to induce an alteration of the function or the structure of a neural component. For instance, plasticity can be induced by an external stimuli, an internal state, a lesion, etc. Such alterations of neural components occur across a large range of spatial scales and take place through different mechanisms: i) intrinsic plasticity relates to the continual alteration of a neuron's inherent biophysical characteristics by neuronal activity [2] ii) synaptic plasticity relates to the activity-dependent modification of the strength or efficacy of synaptic transmission at preexisting synapses [1] iii) representational plasticity relates to the re-organisation of distributed responses as a result of persistent external changes [3]. On the temporal scale, short-term plasticity occurs on the subsecond to minutes time scale in response to an external event such as, for example, an external stimulation and may be transient. Long-term plasticity occurs on the hours, days or years time scale and encompasses long-lasting changes such as the modification of behavior, the formation of new memory, development of new connections across regions, etc.

Across these different spatial and temporal scales, plasticity operates via different means and there are two main forms in which plasticity manifests itself: functional and structural. Functional plasticity involves changes in synaptic strengths without any change in the anatomical connectivity between neurons [4]. This can be realized through the insertion or the removal of synaptic receptors, change in the presynaptic release of transmitters or change in the thickness of the synapse and has been studied in a large number of works $[5,1]$. Structural plasticity refers to changes in the physical anatomical connections (excluding the simple insertion/removal of synaptic receptors). There are numerous evidences of structural plasticity: from the excessive axonal branching during ontogeny with ensuing pruning to the rapid increase in synaptic density during infancy with subsequent slower synapse elimination to adult neurogenesis in the mammalian hippocampus $[6,7,8,9,4]$. Major structural reorganisation of the primary somatosensory cortex has also been observed following amputation or sensory deprivation [3] in primates while the trimming of whiskers in juvenile rats have been shown to induce a realignment of the dendrites of stellate neurons in the barrel cortex [10]. Such evidences suggests that a change in neural activity may induce structural plasticity. The mechanisms underlying plasticity and its relationship with behaviour, learning and memory have been studied for several decades $[11,12$, 13] with a major focus on functional plasticity more than structural plasticity $[14,15,16]$. For example, long term potentiation, long term depression and spike time dependent plasticity have been intensively explored experimentally and widely incorporated in a number of computational works $[17,18,19,20,21]$. However, with the advent of new experimental techniques, such as diffusion tensor imaging, the number of studies in structural plasticity has increased [22] and several of these studies from the last decade have clearly demonstrated the importance of structural rearrangements [23]. It has been widely discussed that structural plasticity confers several advantages, such as improving energy efficiency of network formation, increasing information capacity, amongst others (discussed further in section 4) [24, 25, 26]. Moreover, it holds the capacity to improve learning in a circuit, while conserving resources [27]. This leads us to explore the role played by such structural plasticity in learning and development. While there are several instances across species, from ontogeny to seasonal plasticity (discussed in section 2 and 3), where the role played by such plasticity can be deduced based on the hypotheses prevalent in literature, benefits specific to a particular circuit and function may emerge in certain ecological contexts. Using this particular case of sensorimotor learning in the vocal learning circuitry of birds, where the function fulfilled by structural plasticity is not immediately evident, we inves- 
tigate if structural plasticity can additionally contribute directly to circuit function.

In this article, we propose to review current evidences for structural plasticity and their significance from a computational point of view. More precisely, we aim at investigating if, and how, structural plasticity is critical for vocal learning in juvenile songbirds. To do so, we start by reviewing evidences for structural plasticity across species and categorizing them along the spatial axis as well as along the time course of development. We, then, proceed to draw a parallel with songbirds, showcasing similar phenomenon observed therein. We introduce the vocal learning circuitry in songbirds, as an important example of structural plasticity, and use this specific case to explore the possible contributions of structural plasticity to computational models. Finally, we discuss properties that can be exploited computationally, and unexplored questions which are raised by such models.

\section{Structural plasticity}

Structural plasticity ${ }^{1}$ pertains to the physical changes in neuronal connections. It comprises of changes in synapse numbers, axonal fibre densities, axonal and dendritic branching patterns, synaptic connectivity patterns, and even neuronal cell numbers [4]. Structural plasticity can be categorised according to the spatial scale (e.g. synaptic, axonal, network), the temporal scale (e.g. sub-seconds, minutes, days) and the development period (e.g. sensitive period, childhood, adulthood). Depending on the development period, we can further categorise it based on the cause for structural plasticity: hormonal, learning, pathological, injury-induced.

\subsection{Spatial scale}

Structural plasticity occurs across the whole range of cerebral scales through morphological changes, such as enlargement, growth or apoptosis, that target spines to axon terminals to glia [23]. Recent breakthroughs in recording techniques such as in vivo confocal microscopy have made it possible to gain deeper knowledge onto the underlying mechanisms.

Synapses go through an exuberant growth during early brain development and most of them will permanently disappear as a result of a competitive process involving neurons, synapses and neural growth factors [28]. It has further been observed that lesions in adult brains can lead to an alteration in the synaptic connectivity patterns, due to structural plasticity [29]. Besides sprouting and pruning, structural plasticity can also manifest through neuro-degeneration, neurogenesis [5] and synaptic rewiring, i.e. modification of existing connections, e.g. dendritic spines or axonal branches, by dissociating a pre/post synaptic element, and later, linking it to a different target [30, 31].

Dendritic spines are both highly motile and transient structures. For example, in the barrel cortex, about two thirds of the spines remain for less than a month while some of them appear and disappear within a day [30]. It has been observed in mice that spine turnover and stabilisation can also correlate with learning and memory consolidation, respectively [32]. In addition to their motility, dendritic spines occur in a wide range of size, shape and organelles, which in turn potentially affects different functional properties such as, the synaptic strength, its stability, the postsynaptic receptors, etc [23]. Morphology does indeed have a direct impact on the functional role, with large spines helping to form stable connections, and the transience of thin spines being an aid in learning, for instance.

\footnotetext{
${ }^{1}$ Before diving into the details of the song-learning circuitry, we first need to briefly review generic concepts and principles related to structural plasticity. The knowledgeable reader can skip this section and head directly to section 3.
} 
Axon arbor structure can be manipulated within a circuit-level mechanism of learning. In macaque monkeys, trained to perform a contour detection task, Van Kerkoerle et al. [33] found that there is an extensive sprouting and pruning of axonal collaterals in cortical regions which correspond to the trained area of the visual field. In addition to neurogenesis, neurons have the capacity to degenerate part of their axon in order to refine connections through axon pruning or more drastically, cell apoptosis [34,6]. Both, the mechanisms underlying such formation of short-range or long-range axonal connections as well as the selective degeneration of unoptimal axonal branches could potentially have long-term impacts on circuit function [35].

At the network level, significant structural changes occur that affect large cerebral regions. For instance, in blind Braille readers, representation of the reading fingers in the somatosensory cortex is larger than nonreading fingers or any finger of non-Braille readers [36]. Similarly, it has been shown that a massive reorganization of the motor cortex occurs within a few hours following the transection of the facial nerves in the rat [37]. There is, thus, overwhelming evidence that structural plasticity serves a functional role by manipulating various elements of the central nervous system, from spines to synapses to axonal arbors to neurons. We won't detail adult neurogenesis since it is beyond the scope of this review, but we redirect the reader to the studies by Gould [38], Paredes et al. [39] and Pytte [40].

\subsection{Time course across development}

Structural plasticity can be initiated by several factors at several timepoints in one's lifespan. During development, dendritic spines exhibit structural plasticity, by variable sprouting and pruning, depending on experience. Synaptic turnover is extremely high during this period, and a majority of newly sprouted dendritic spines in the mice somatosensory cortex, are lost, within the span of a mere few days [41]. As development of an individual progresses, the course of puberty also marks some structural changes. The beginning of higher rates of pruning of dendritic spines in the human frontal cortex, and cortical thinning in humans have been shown to correspond with pubertal development [42].

During development, structural plasticity is limited in time by what is known as 'sensitive periods'. These periods designate limited periods of time wherein several connections or skills are modified permanently and significantly, based on the information acquired through experience [43]. Sensitive periods occur in the prenatal brain and continue throughout development but are very limited during adulthood. For instance, newly hatched chicks memorize the characteristics of the first moving object they encounter, and subsequently show a preference for it. This "imprinting" behavior can only be acquired within the first few days following hatching, and involves age-dependent remodeling of neural networks in the visual and associative areas of the chicks' brains [44]. In humans, language acquisition is, often, a highly cognizant and arduous task for adults, as compared to the relative passive learning by children. It can be argued that, before the end of puberty, certain neural circuits are more susceptible to assimilating such skills [45]. A prominent study by Hubel and Wiesel [46] found that closing one eye of kittens, but not of adult cats, causes the permanent loss of visual responsiveness of neurons in the primary visual cortex to stimulations to the deprived eye (ocular dominance plasticity). Moreover, axonal sprouting and branching of thalamic neurons is curbed by visual deprivation during development in rodents, and retinal ganglion cells extend their receptive fields in turtles which have been raised in the dark $[4,47]$. Hence, while the beginning and end of sensitive periods are triggered by molecular signals delivered timely during development, sensory experience is crucial and can modulate the opening and closing of sensitive periods, especially during development $[43,48]$. 
Beyond development as well, structural plasticity has an impactful presence throughout the lifespan of an animal, triggered by various reasons, ranging across hormonal, lesion-induced, pathological and training. Hormonal changes lead to structural changes in neural circuits. Structural changes in the circuitry is crucial for the seasonal control of reproduction. Morphological rearrangements cause seasonal inhibition of a certain hormonal secretion in adult ewes [49]. Similar hormonal changes can be internally triggered, as in the previous case, or caused due to external chemical influence. Administration of drugs, such as, amphetamine or methylphenidate alters the organization of dendrites in the prefrontal cortex, causing reduced play initiation, as well as impaired working memory functioning [50]. Furthermore, lesions or adverse conditions can induce changes in the network. Lesioning a part of the retina leads to an adjustment in the cortical topography, by causing the receptive fields of cells in the cortical scotoma to adapt to representing the retinal area surrounding the lesion [37]. Pathological conditions can cause major upheaval in the neuronal networks. Uncharacteristically higher spine densities has been observed in certain parts of the frontal, parietal and temporal lobes in patients with Autism Spectrum Disorder [51]. Continuous exposure or targeted training can also potentially cause structural changes. The difference in proficiency, between professional musicians, amateur musicians, and non-musicians, in discriminating tones has been traced to certain structural differences, including the higher gray matter volume in the left Heschl's gyrus in musicians [52]. Structural plasticity clearly plays a sensitive and functional role in several processes during and after development.

\subsection{Interaction between structural and functional plasticity}

The previous section has demonstrated the ubiquitous presence of structural plasticity across species and across developmental and spatial scales. Structural plasticity encompasses a variety of mechanisms that depends on the level under consideration; the processes underlying spine turnover are very different from the ones that drive axon arborisation [53, 54]. However, such processes of structural plasticity do not occur in isolation. We find abundant evidences wherein structural plasticity interacts with the other various forms of plasticity within neural circuits. As discussed in section 1, plasticity in neural circuits ranges from the alteration of the intrinsic biophysical properties of a neuron, and the experience-dependent modification of the efficacy of synaptic transmission, to a massive overhaul of axonal connections within and across brain regions. Inducing synaptic plasticity not only affects the generation of action potentials by a neuron but also affects the intrinsic properties of a neuron, such as synaptic integration. For instance, induction of long-term potentiation in slices of CA1 pyramidal and purkinje neurons from the rat hippocampus and cerebellum, respectively, has been shown to have strong effects on the intrinsic excitability of a neuron as well as dendritic integration [55, 56]. Conversely, alteration of neuronal excitability, by blocking after-hyperpolarisation (AHP), enhances induction of long-term potentation in the hippocampal pyramidal neurons [57]. Reduced AHP of pyramidal neurons in the rat piriform cortex, following operant conditioning, also has been linked with improved learning capacity [58]. Similarly, functional and structural plasticity are tightly inter-connected. Initiation of structural plasticity may be triggered due to an external lesion, or by an internal hormone release [16]. In this wide landscape, there is an interesting case where structural plasticity is driven first and foremost by neural activity. De Roo, Klauser, and Muller [31] observed an increase in spine turnover following induction of long term potentiation in slice culture of neurons. Further, Oh, Hill, and Zito [59] showed an activity-dependent shrinkage of dendritic spines. It has been also observed in hippocampal slice cultures that stimulation led to an increased stabilisation of stimulated synapses and faster pruning of non stimulated synapses [23, 60]. On the other hand, such synaptic pruning can lead to an increased inhibitory nature of the circuitry, which can have varied impacts. For instance, the late development of an inter-neuron subset in the primary visual cortex causes the maturation of the inhibitory circuitry which plays 
a crucial role in the opening and closing of the sensitive period [43]. Thus, we can see that structural and functional plasticity have a strong mutual interaction, and are both closely related to the neural activity of the related network. While, in the former examples, a functional change is able to trigger structural plasticity, in the latter case, structural plasticity shows a prominent functional impact. At a larger scale, the increase in inhibitory activity curtails the generation of new synapses and the elimination of existing ones, which ultimately leads to the stabilisation of the circuitry [61].

These observations suggest that certain instances of structural plasticity stem from functional changes, and in turn, have the potential to induce a prominent functional impact. It seems more than relevant to investigate the interactions between structural plasticity, neuronal activity and the function of the related networks. Given the complex interactions between network structure, activity and function, theoretical investigations in computational models will likely be required to shed light on the underlying mechanisms. Structural plasticity has been sporadically incorporated into computational models (see section 4). We hypothesise that via such interactions, structural plasticity could potentially fulfill, not merely an auxiliary role in the functioning of a circuit (e.g. energy optimisation), but also a more direct role towards facilitating the intended function of a circuit. We will advocate below that vocal learning in songbirds is a useful paradigm to understand the implications of structural plasticity on the organization of neural networks during development and beyond, on the network function and the underlying neural code, and ultimately on behavior.

\section{From structure to neural activity to song}

Firstly, we shortly review the analogies between birds and mammals, in both a behavioral and neuroanatomical sense, and touch on the evidence accumulated in support of the hypothesis that the two taxa share general principles in the organization of brain networks, the constraints bearing on their construction during development and re-organization following injury or natural remodeling of brain circuits. Whether these common principles are conserved, or the result of convergent evolution, is beyond the scope of our review (see [62]). Once the parallels have been established, we will highlight that structural plasticity is ubiquitous in the bird brain, just as it is in mammals. We will then focus our survey on the brain circuits involved in the acquisition and production of complex vocalizations in songbirds, where the relation between neural structure, activity and function has been best studied. To this end, we briefly describe the vocal learning circuitry, then delve into the details of structural plasticity in this circuitry and finally extract computationally useful properties herein. As we will focus our review on structural changes undergone by the song-related network across development and adulthood, we deliberately leave aside the likely crucial role of functional plasticity in song acquisition and maintenance, discussed elsewhere [63, 64].

\subsection{General behavior and brain anatomy in birds}

The successful evolution of mammals in diverse ecological contexts is thought to rely, at least in part, to the evolution of their large brains, conferring them with behavioral flexibility and cognitive abilities and making them efficient predators and competitors. These cognitive abilities are diverse and include (but are not limited to) complex sensorimotor coordination and adaptation, memorization, planning and anticipation capacity, analytical and deductive reasoning, sophisticated social interactions, and introspective judgement. While many of these complex cognitive skills have first been thought to be the hallmark of humans, or exclusively present in mammals gifted with large brains, abundant experimental evidence now demonstrates that several bird 
species also display many, if not all, of the same cognitive functions as mammals [65, 66]. Sophisticated sensorimotor learning abilities are demonstrated in songbirds by song acquisition, with remarkable parallels to human speech learning, including its reliance on high auditory processing of auditory feedback, tight coordination of vocal muscles and social interactions [67, 63]. Various bird species display food caching behavior and rely on months-old spatial memory traces to retrieve their spread-out cached food [68], illustrating their excellent episodic-like memorization and planning capacities. Tool use and other problem-solving tasks have been successfully solved by several bird species, many from the corvidae family [69]. Many species of birds, including starlings, crows and parrots, have been found to maintain complex social organization among colonies of thousands (and more) of subjects [70, 71], relying at least partly on vocal communication. Finally, parrots display cognitive abilities that we have until now considered unique to humans and other primates (conceptual representation, combinatory learning, counting [72]) and magpies can recognize themselves in a mirror [73], a skill thought to require a representation of oneself and only sparsely present in mammals.

Altogether, the similar cognitive abilities of birds compared to mammals suggest that similar constraints apply to the organization of their nervous system to optimize their behavior. Studying the emergence of brain function during development or re-organization in adulthood in birds is, thus, just as promising as it is to rely on mammalian animal models.

The complex behaviors displayed by birds and briefly summarized above rely on brain circuits that display, for the most part, major biochemical, anatomical, and physiological differences as compared to their mammalian counterparts, but still remain surprisingly analogous in their functional organization $[62,66]$. Basic avian brain anatomy already differs from that of mammals, the most notable discrepancy being the absence of a laminated neocortex in the avian telencephalon [74, 75]. Rather, the avian pallium is inherited from the reptilian Dorsal Ventricular Ridge and is organized into a largely continuous field of nuclei. For this reason, the avian telencephalon has long been considered to mainly consist of a hypertrophied basal ganglia structure (or paleostriatum, see [76,77]). While this organization appears very different, the nuclei of the avian pallium show similar connectivity, neuronal types and functional properties to those of the mammalian cortex, amygdala, and claustrum. Within this area, birds clearly display a hippocampus, piriform cortex and olfactory bulb. The hyperpallium in birds (previously/also called the Wulst), also included in this area, displays a strong analogy with the primary visual area and a primary somatosensory area of the mammalian cortex, in terms of thalamic input, connectivity, and electrophysiological properties [78]. The Nidopallium contains various auditory areas that display a similar functional organization as the mammalian auditory cortex, although the spatial arrangement of the various network components is very different in birds [79, 80]. At a finer scale, comparative developmental analysis and neurochemical data reveal a surprising extent of similarities in the neuronal subtypes and among birds and mammals [81, 82].

In the ventral part of the avian telencephalon, 'subcortical' structures are older from an evolutionary point of view as compared to the pallium, and therefore share even more similarities with mammalian subcortical structures. In particular, the avian brain contains homologues of the mammalian septum, basal ganglia (BG) and several other nuclei, as unveiled by developmental, topological, neurochemical, cellular, connectional and functional data [83]. Moreover, a modern revision of the avian anatomical nomenclature has now provided a common language for studying the function of the avian subcortical nuclei [77].

Concerning the BG, there is an avian circuit that has been looked deeper into: the song-related BG-thalamo-cortical loop of songbirds. Indeed, songbirds have specialized a portion of their forebrain-BG circuitry expressly for the purpose of song learning. Recent advances in anatom- 
ical, physiological and histochemical characterization of avian BG neurons and circuitry have revealed a homology between this circuit and the mammalian motor loop of the BG-thalamocortical network [84, 76]. In particular, the song-related BG nucleus Area X differs from mammalian BG in its gross anatomical structure, but it displays surprisingly similar circuitry at a finer scale. It is embedded within a region homologous to the mammalian striatum and receives a particularly strong dopaminergic projection [85]. It contains neuron classes corresponding electrophysiologically and morphologically to those in the mammalian striatum [86, 87]. In addition, Area X contains a class of neurons with pallidal properties, which directly project to the thalamic nucleus DLM [88, 89]. We will focus below on the song-related circuits in songbirds, including this BG-thalamo-cortical loop.

Given the strong homology (for subcortical structures) or analogy in the organization of brain circuits driving complex behavior between birds and mammals, it is most likely that the functional organization of these circuits rely on the same basic principles. Therefore, studying the computational advantages of structural plasticity in birds will improve our understanding of this basic principle and provide generalizable theories for how development and remodeling acts to (re)shape brain function. Now, we will discuss the specific case of the vocal learning circuitry in songbirds.

\subsection{Avian vocal learning}

For context, we begin by providing a brief overview of the vocal learning process in birds, along with the related anatomy.

\section{Song acquisition and production}

Songbirds use learned vocalizations to communicate during courtship or aggressive behaviors. Akin to speech learning in humans, vocal learning in young birds requires the coordination of vocal muscles to reproduce previously experienced adult vocalizations. Singing is a sensorimotor skill and song acquisition in juvenile birds is also highly dependent on hearing the adults they will imitate, as well as themselves as they practice, and this dependence wanes as the birds mature. Strikingly, the gene FoxP2, linked to speech learning in humans [90], is also implicated in avian song learning [91]. Similar neural mechanisms underlying vocal learning are most probably involved in humans and birds [67], and the study of these mechanisms in birds could shed light on the neurobiological bases of speech learning.

Interestingly, in birds, as in humans, imitative vocal learning is characterized by several phases [67]: (i) the sensory phase enables human infants or juvenile birds to build a neural representation of adult vocalizations, which will guide later vocal production; (ii) during the sensorimotor phase the young subjects start to vocalize, initially producing babbling sounds and then adapting its vocal output to imitate previously heard vocalizations; (iii) finally the produced vocalization becomes more and more stereotyped and vocal plasticity significantly drops. This final phase is called crystallization in birds. Each of these phases is bounded by a sensitive period [63]. In most bird species, if the young subjects have not experienced conspecific adult vocalizations before a species-specific age limit (e.g. 60 days post hatch (dph 60) in zebra finches, around 2 years in children), imitation will be virtually impossible because the sensory phase of vocal learning is closed past that age. Similarly, the sensorimotor phase closes with puberty in many species (around dph 90 in zebra finches, 12-14 years in humans) when vocal exploration drastically decreases (and vocalizations become more stereotyped), making vocal imitation more difficult and reducing greatly the vocal plasticity. Seasonal birds may re-open the sensorimotor phase yearly and therefore display cyclic sensitive periods locked to seasonal changes. 


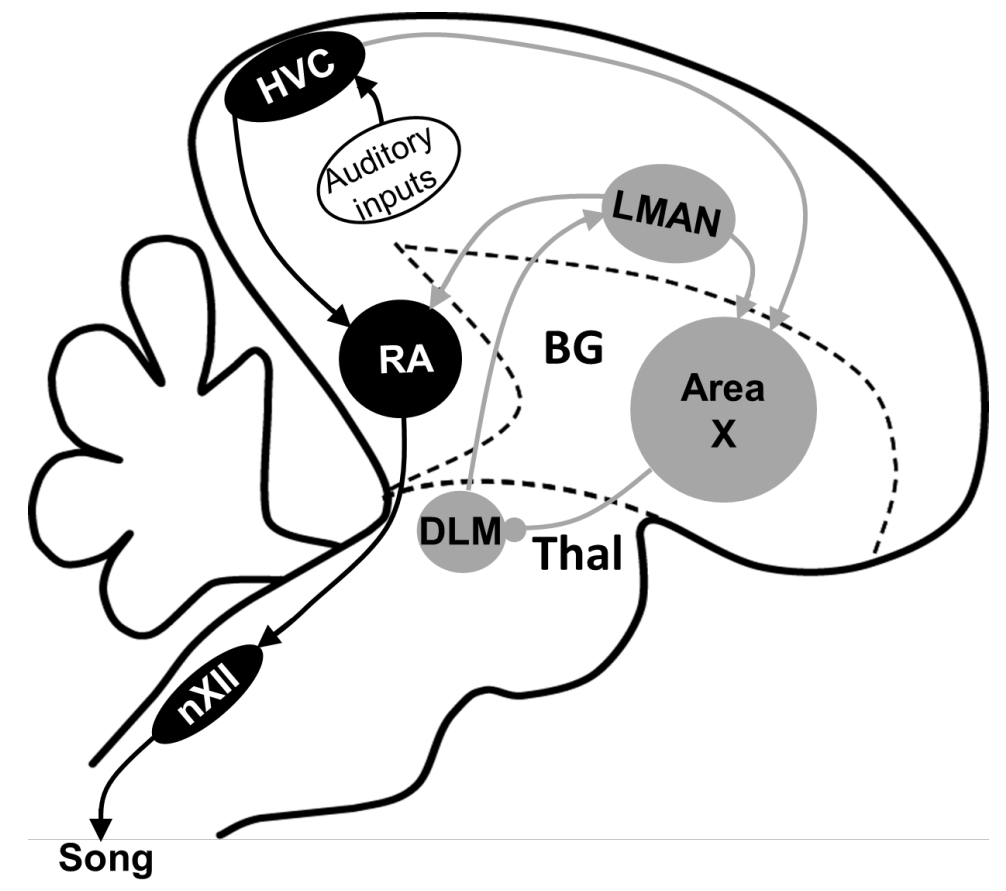

Figure 1: Schema of the neural substrates involved in vocal learning in zebra finches. The cortical pathway, shown in black, comprising the HVC and RA, is primarily responsible for vocal production. The anterior forebrain pathway (show in grey), consists of the LMAN, Area X (BG homologue) and DLM (thalamus homologue) and plays a crucial tutor role in vocal learning.

These sensitive periods and the underlying phases of vocal learning are driven by physiological signals that constrain the development of neural circuits in the central nervous system, and thereby affect the behavioral plasticity. What are the changes undergone during vocal learning by the related neural circuits? Can we understand the various behavioral phases in light of circuit changes? In other words, how does structural plasticity related to the development of brain circuits underlying vocal learning affect the imitation process? We will illustrate below how behavioral, anatomical, neurochemical and electrophysiological data collected in songbirds (mostly in zebra finches) may help bridge the gap between structural plasticity and behavior.

\section{Anatomy of the song system}

In birds, the sensorimotor skill of song production and its learning has a dedicated set of interconnected brain nuclei collectively known as the "song system" (see Figure 1), that ultimately coordinate the patterned breathing and vocal muscle activity necessary for vocalization. This dedicated circuit makes the songbird an outstanding model to study the neural mechanisms of vocal learning and more generally, the function of neural circuit in sensorimotor learning.

The song system includes a 'motor pathway' that is required throughout life for normal song production, and a BG-thalamo-cortical circuit necessary for song learning, plasticity and maintenance, called the 'anterior forebrain pathway' [92]. The song system receives auditory information through the projections from the high-level auditory areas Field L, NCM (caudo-medial nidopallium) and CM (caudal mesopallium), and the pallial regions analogous to the auditory cortices of mammals (see section 3.1), to the nucleus HVC (used as a proper name) [93].

The motor pathway includes the premotor cortical nucleus HVC and the robust nucleus of the 
arcopallium (RA), which is functionally equivalent to the laryngeal motor cortex. The HVC is involved in generating the timing and sequencing of song [94, 95]. RA receives inputs from the HVC and projects directly to respiratory centers and to the brainstem motor neurons controlling the vocal organ; the respiratory centers send recurrent information back to the HVC via the thalamus, reflecting the importance of bidirectional coordination between telencephalic and brainstem structures in vocal control $[96,97]$.

The BG-cortical loop indirectly connects the HVC and RA, and like the cortico-basal ganglia circuitry in mammals, plays a crucial role in motor learning. It consists of three nuclei connected in a loop: the BG nucleus Area X, the medial portion of the dorsolateral thalamic nucleus (DLM), and the lateral magnocellular nucleus of the anterior nidopallium (LMAN). This loop is closed in the macroscopic sense (i.e., the projections form a recursive loop). The LMAN - Area X, Area X-DLM and DLM - LMAN projections within the loop are topographic [98, 99], thus demonstrating that this BG-cortical loop is also microscopically closed. The song-related BG nucleus Area $\mathrm{X}$ receives strong dopaminergic innervation from the midbrain [84]. Because this specialized cortico-BG circuit is discrete and devoted to a specific well-defined and naturally learned sensorimotor task rather than a broad range of motor behaviors, it is particularly tractable for elucidating the interwoven sensory, motor and reward signals carried by BG, and the function of these signals in skill learning and execution [83].

During song acquisition, the integrity of the song-related BG-cortical loop is required for proper imitation of adult vocalizations $[100,101]$. Although its precise role in song learning has long remained mysterious, recent experimental studies have shed light on its function during learning. Multiple lines of experimental evidence support a role of the BG-cortical loop in driving and modulating acoustic variability in song. In particular, lesions or inactivations of the cortical output of the loop (LMAN) reduce the acoustic variability of plastic song in juvenile birds [102, 103] and changing dopaminergic input to the circuit triggers changes in song variability [104]. The song-related BG circuit also guides changes in motor output by generating an error-reducing motor bias that is rapidly incorporated into the cortical premotor network [105]. In birds, as in mammals, it is believed that the adaptive motor changes elicited by the BG are learned through a reinforcement learning mechanism. Accordingly, dopamine delivery in the BG provides an online evaluation of song quality [106] analogous to reward prediction errors signaled by dopamine during conditioning in mammals [107]. This dopaminergic signal provides a reinforcement signal that drives error correction during learning $[108,109]$. The dopaminergic input is known to modulate synaptic plasticity in birds [110], akin to mammals [111]. Thereby, the BG output implements behavioral adaptations guided by dopaminergic reinforcement signaling to optimize motor output. These behavioral adaptations are ultimately consolidated in cortical networks following extensive training $[105,112]$.

The whole network of brain nuclei involved in song production in songbirds - the song system offers a great model for studying brain circuits and their role in behavior. The numerous genes implicated in human diseases that are enriched in the song system nuclei and exhibit differential expression, both at specific points in development and during behaviors, such as listening to song or singing, also opens up interesting paths for the study of pathophysiological processes $[113,114]$. We argue that studying the benefits and consequences of structural plasticity in this system, through a functional standpoint, may shed light on the general principles that make structural plasticity advantageous for the central nervous system in the perspective of learning complex skills. 


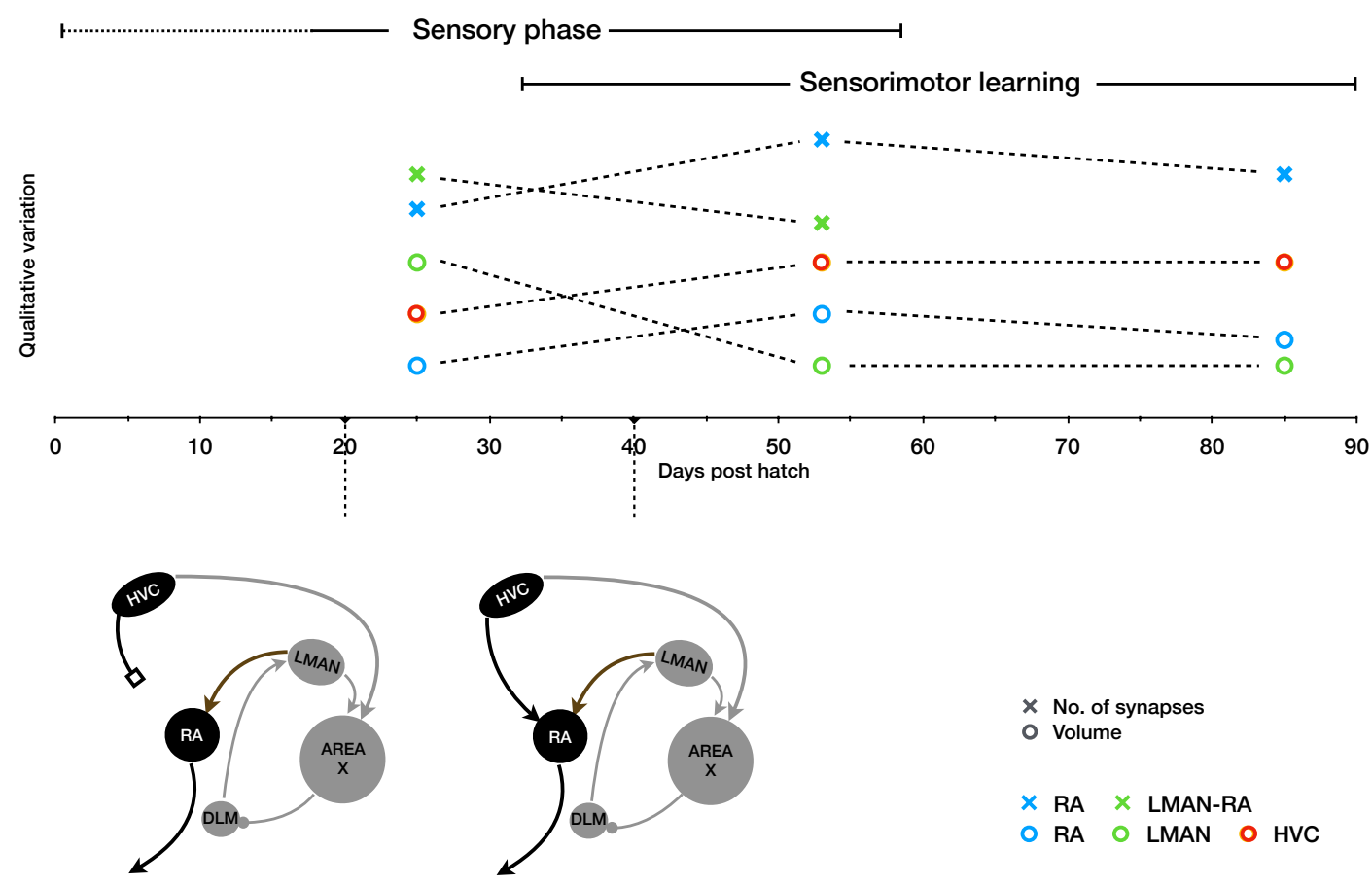

Figure 2: Timeline of development of the vocal learning circuitry in zebra finches $[115,116$, $117,118,119,120,121,122]$. The bottom panel shows the axons from HVC entering the RA, to form the cortical pathway responsible for vocal production, much after the anterior forebrain pathway (theorised to provide a tutor signal for vocal learning) is completed (bottom panel). This is accompanied by significant changes in the neural regions involved. During the initial sensorimotor period, the volume of the RA and HVC increase with a significant decrease in the LMAN volume, while the synapses within the RA increase, both from HVC axons and from RA interneurons. In the later stages of the sensorimotor learning, decreasing song variability is accompanied with a stabilisation of the volume of the LMAN and HVC and a slight decrease in RA volume. 


\subsection{Structural plasticity in songbirds}

Similar to mammals, birds display plasticity from the scale of dendritic spines to synapses to networks throughout their lifespans, both during development and in adulthood. Internal factors (such as the concentration of circulating hormones) and external factors (such as daily light cycles, but also injury or lesion), can trigger plasticity in birds. For instance, the avian olfactory pathway which projects into paralimbic areas, before hatching, is displaced competitively by the development of visual, auditory and motor pathways after hatching, as a result of higher audio-visual exposure [123]. Neurogenesis and circuit reorganization is also common in adult birds. The hippocampus undergoes seasonal neurogenesis and reorganization in food-storing birds and brood parasites [124], and higher exposure leads migrating species (passerines) to undergo more neurogenesis in areas that process spatial information, than non migratory species [125]. Interestingly, similar mechanisms underlie adult neurogenesis in both avian and mammalian species [97]. The brain circuit where structural plasticity has been most studied in birds is arguably the song system in songbirds [126]. In this section, we provide a short review on the current evidence for structural plasticity in the vocal learning circuitry in birds.

\section{During development}

Song acquisition happens in juvenile birds while their brain is still in development. As a consequence, the song-related neural circuits are still in the process of being built and there are many forms of structural plasticity in the network, during the song learning period.

Auditory experience may already have an influence on brain development before hatching [127] and the auditory pathway from the ears to high auditory areas in the pallium is built early in development. The anatomical structure of the auditory network is thus largely mature in young birds when the sensory learning phase starts (dph 20), with all major anatomical connections being present. Exposure to adult vocalization, at that stage, shapes the auditory responses of neurons in the high-level auditory nuclei of the pallium where song-selective responses occur relatively early (before dph 35) [128]. Receiving strong afferents from these auditory nuclei, neurons in HVC and its efferent structures show a progressive emergence of song selectivity during the sensory period of song learning [67]. Chen and Meliza [129] demonstrate that during development, there are significant changes in the phasic excitability in the caudal mesopallium $\mathrm{CM}$, a cortical auditory region which exhibits selective responses to familiar conspecific songs. Exposure to tutor song has been shown to induce cell-type specific changes within the ion channel expression patterns of HVC neurons, depending on whether they project to the RA or the Area X. These changes happen at a magnitude that can potentially alter network function [130]. Moreover, daytime exposure to tutor song had profound effects on the distributions of inter-spike intervals of RA bursting activity during sleep, depending on the tutor song [131]. Deafening adult birds, on the other hand, decreases dendritic spine size and stability of Area X projecting HVC neurons, along with an increase in mean spontaneous action potential firing rates and decrease in inter-spike intervals [132]. Daou and Margoliash [133] investigate the intrinsic properties of Area X projecting HVC neurons further, and discover that birds displaying good copies of their tutor's song exhibit similar spike morphology, spike trains, and ion current magnitudes, indicating that these intrinsic dynamics may be molded by auditory and vocal experience. Additionally, considering conditioning using distorted auditory feedback also elicits a prominent effect on the intrinsic properties of HVC neurons in adults, it has been suggested that such intrinsic plasticity could form an alternate/complementary mechanism for learning via their effect on synaptic efficacy and network interactions [133, 134]. Further, functional plasticity is likely involved in the shaping of auditory responses in this network [135]. The synaptic rewiring in HVC is also evidenced by a large turnover of dendritic spines early in the sensory learning phase, followed by a 
rapid stabilisation of spine dynamics [136]. This correlation of circuit changes with tutoring may indicate a functional role of structural plasticity in sensorimotor learning. On the other hand, with development, spine turnover in the HVC decreases in untutored birds too. Hence, this stabilisation might potentially underlie the closing of a sensitive period, where they are more receptive to learning behavioural change. The underlying cellular and molecular mechanisms triggering the changes in plasticity are still under investigation. Interestingly, perineuronal nets surrounding groups of neurons and limiting axonal and dendritic processes could mediate changes in plasticity [137]. Further work is however required to confirm their causal role in the opening and closing of sensitive periods.

Contrary to the ascending auditory pathway, the song system is highly immature when birds start the song learning process, and structural plasticity is ongoing both during the sensory and the early sensorimotor period of song acquisition (Figure 2). Among the two pathways of the song system, the BG-cortical loop develops first and is fully formed by dph 20, before the sensorimotor phase begins [122]. Indeed, in juvenile male zebra finches, at the onset of song learning, axonal terminals from LMAN enter Area X (dph 20), Area X terminals enter DLM by dph 20 [121], and DLM terminals enter LMAN before dph 15 [120]. This BG-thalamo-cortical circuit is topographic in nature and reaches maturity before dph 20. $[98,138]$. Concerning the input pathway of the BG-thalamo-cortical loop, HVC neurons projecting to Area X innervate this target by dph $20[139,118]$. The output pathway of the loop is also formed early, with LMAN projections entering the RA by dph 15 [119]. The number of synapses made by LMAN axons onto RA neurons decreases substantially (-70\% between dph 20 and 60 ) over the course of vocal development [119]. While anatomical connections between the nuclei are stable from dph 20 to adulthood, the nuclei of the BG-cortical pathway nonetheless experience dramatic volume changes. Between $20 \mathrm{dph}$ and $60 \mathrm{dph}$, LMAN volume and neuron number decline by $50 \%$, while the volume of Area $\mathrm{X}$ increases by $50 \%$, due to neurogenesis $[140,118]$.

The motor pathway, on the contrary, develops very late in the song learning process. First, both HVC and RA grow slowly, reaching adult size only after dph 40 and dph 70, respectively [117]. In HVC, the number and size of neurons both increase dramatically [116]. Meanwhile, the synapses in the RA, from the HVC and LMAN are drastically rearranged at the onset of the sensorimotor phase. HVC axons reach the dorsal border of RA by dph 15, but, unlike the LMAN, they form synapses with the RA only between dph 30 and $40[119,122]$. In the sensorimotor period, between dph 20 and 60, the reduction of synapses between the LMAN and RA is accompanied by a tripling of the number of synapses within the RA and an increase of the RA volume [119, 116, 117].

The most striking consequence of the late development of the song system is that the motor pathway, which drives adult vocalizations, does not contribute to the babbling vocalization, called subsong, produced by juveniles in the early sensorimotor phase of song learning [141]. At that stage, the HVC-to-RA pathway is not yet functionally strong enough to drive singing and LMAN projections are driving subsong-related activity in RA. During the following days, the formation and strengthening of inputs from HVC to RA leads to the emergence of plastic song, driven both by LMAN and HVC $[119,142]$. As learning progresses, the influence of the LMAN inputs to the RA reduces due to the strengthening of the synaptic connections from the HVC. Further, the variability of the song decreases with development and practice (dph 60-90), along with a significant pruning of the HVC inputs to the RA, albeit with a strengthening of the surviving connections [142]. 


\section{During seasonal plasticity}

Post-development, hormonal changes and external factors continue to induce structural changes in the brain in many vertebrates beyond the developmental period [143]. Most temperate songbird species breed seasonally and display a pronounced seasonal plasticity in their singing behavior [144]. The song initially learned as a juvenile undergoes a pattern of yearly changes triggered by changes in the light cycle and mediated by sex steroid signaling in the brain $[145,146]$. Producing highly stereotyped songs during breeding, birds stop singing during the summer molt, resume singing in fall with short-duration songs of variable structure at lower rate and volume and gradually sing longer, louder and more stereotyped songs until the next breeding season $[147,148]$. These changes in song are accompanied by a large structural reorganization of the neural circuits in the song system [149], auditory areas [150] and beyond [151]. This naturally occurring plasticity in the songbird brain is perhaps the most pronounced observed in any adult vertebrate. The volume of many song-related brain regions, as well as their number of neurons and synapses, increase dramatically in anticipation of the breeding season [147, 126], while the physiological and functional properties of their neurons are altered $[152,153]$. The entire volumes of several song nuclei, including HVC, RA and Area X, are considerably larger during the spring breeding season than during autumn and winter in seasonal birds [154]. The increase in volume may reflect an addition of new neurons through neurogenesis, notably in the HVC [155, 156], or dendritic growth and synaptogenesis, with an increase in the number of dendritic spines, as seen in the RA or Area X [157]. Meanwhile, neuron number in LMAN and their inputs to RA remain relatively constant [158]. Altogether, it is interesting to note that the strengthening of the HVC-RA pathway before the breeding season recapitulates at least in part the late development of the motor pathway during song acquisition in juveniles. Interestingly, seasonal plasticity is regulated by the same endocrine signals as juvenile song learning $[159,160]$ and may therefore exploit similar mechanisms as those acting during early ontogeny [161].

\subsection{Functional impact of structural plasticity}

There are parallels in the manifestation of structural plasticity between mammals and birds. Insights drawn from the songbird literature can be used to understand neural circuitry and behaviour in mammals, as well. They present an avenue to explore the contributions of changes in neural circuitry beyond functional plasticity.

Certain fundamental elements of structural plasticity can potentially confer benefits when incorporated into computational models. During song learning, there is an early excessive sprouting of neurons in HVC and RA, followed by a significant pruning of the HVC-RA synapses, and strengthening of the surviving connections [119]. This form of abundant sprouting followed by selective pruning is a commonly adopted technique to model changes in network connectivity. This phenomenon can also be employed to encode the trade off between exploration and exploitation, to represent decreasing tendencies to explore, as learning proceeds [162,142]. Also, spine turnover and terminal size can be useful parameters in modeling the consolidation of learned motor action or behaviour, in lieu of solely modifying synaptic weights [136, 119]. Beyond structural plasticity, it could also be interesting to consider integrating features of intrinsic plasticity within computational models, for instance, by simulating the alteration of intrinsic excitability of a neuron by introducing activity-dependent modifications within the activation functions in typical neural network models, as suggested by Daou and Margoliash [134].

Apart from the general usage of these basic elements to computationally exploit the properties of structural plasticity, there are specific insights that can be drawn from vocal learning in songbirds, where structural plasticity directly has a crucial functional impact on learning. As 
we have seen in the section 3.3, a common feature of the developmental and seasonal structural plasticity in the song system is the progressive strengthening of the motor pathway and its increased synaptic drive on RA neurons compared to the early shaped and steady input from LMAN. Interestingly, in both cases, induction of large variability in the song by LMAN occurs in the condition where HVC inputs to RA are not as prominent [163, 141]. The putative effect of this relative change in the balance of inputs from HVC and LMAN in RA is crucially influencing the activity of neurons in RA and how they mediate changes in song.

Both in the early phase of juvenile learning and during fall in seasonal songbirds, LMAN drives variable patterns of activity in RA, resulting in less structured and more variable songs $[163,102]$. Accordingly, the singing related activity of RA neurons gradually changes from highly variable firing patterns to precise and sparse bursts of spikes locked to song motifs during juvenile learning [164]. LMAN input is necessary for the expression of RA firing variability, and the change from variable to stereotyped firing patterns in RA throughout development could be explained by the strengthening and pruning of HVC inputs to RA (that drive stereotyped patterns) while LMAN inputs remain unchanged [142]. As HVC input to RA becomes larger, it drives stronger bursting in RA interleaved with hyperpolarized periods of silence due to recurrent inhibition [164]. LMAN inputs to RA are mediated mostly through voltage-dependent NMDA receptors $[165,102]$, and its influence on RA firing is weak in this condition. The influence of LMAN on RA is thus diminished, resulting in a progressively more stereotyped song [102]. Interestingly, this scenario does not require any seasonal or developmental modification in the BG-forebrain circuit to see its influence on song strongly modulated. It ensures that LMAN-driven variability is expressed long before the HVC driven temporal structure of the song motif emerges, and likely results in the two-phase learning, including early babbling in subsong followed by temporally structured plastic song.

The strong influence of the LMAN on the RA early in development initially drives subsong and then drives the variability in plastic song; thereafter, HVC plays an increasingly strong role in driving the stereotyped firing of RA as the bird approaches crystallization. The same scenario seems to be recapitulated at least partially during seasonal plasticity due to the regrowth of HVC and the entrance of new axons in RA. Why is HVC input arriving so late in the RA during song acquisition or during seasonal relearning? And why is learning divided in these two phases babbling and plastic song? In the next section, we draw a parallel with optimisation techniques, commonly used in machine learning, to investigate the potential roles of these phenomena in sensorimotor learning.

\section{From biology to machine learning}

We have seen that structural plasticity is shared among different species, occurs (non-uniformly) across the whole lifespan and scales from the level of dendritic spines up to distributed cortical regions. It leads us to question why plasticity of this kind is as prevalent, and if it confers any benefits to the development of neural circuitry. Models of experience-dependent structural plasticity have shown that rules of dendritic and synaptic growth, derived from experimental data [166], improve information transmission in networks with small-world topology (i.e. high clustering coefficients and short path lengths) [167]. Knoblauch et al. [168] simulate structural plasticity by accommodating potential future synapses and find that this leads to a higher storage capacity per synapse than networks with only synaptic weight plasticity. Moreover, Chechik, Meilijson, and Ruppin [24] show that energy efficiency of memory storage, indeed, is improved by using the strategy of excessive sprouting followed by synaptic pruning. Structural plasticity can also influence the development of synaptic connectivity within networks. Poirazi and Mel [169] show that 
memory formation or long-term information storage might also be governed by the co-activation of synapses on a shared dendritic branch rather than merely synaptic weights. Stepanyants, Hof, and Chklovskii [170] complement this study, using geometric analysis to demonstrate that dendritic spines improve information capacity by increasing the specificity of dendritic connections. Sailor et al. [171] further demonstrate the functional role of dendritic arborisation of adult-born granule cell inter-neurons in the olfactory bulbs of adults. Simulations by Spiess et al. [27] show that structural plasticity improves learning by decreasing response noise, and when combined with pruning, also reduces the training time. Further, models such as Lightheart, Grainger, and $\mathrm{Lu}$ [172] incorporate the effect of structural plasticity by using constructive algorithms to simulate network growth which also account for the creation of new neurons. Such comparatively slower structural plasticity could balance the trade-off between stability and plasticity in order to help evade catastrophic forgetting [26, 168]. Knoblauch, Palm, and Sommer [173] further demonstrate that this strategy also helps optimise information capacity in biological networks [174].

Despite these clear advantages of incorporating structural plasticity, it has seldom been used in machine learning and computational neuroscience literature. Most of the time, the architecture of a model is chosen prior to the learning phase and remains fixed until the end. There are of course notable exceptions. One of the earliest and best known example of a computational model taking full advantage of structural plasticity is the growing neural gas model [175] that learns the topology of the input thanks to the dynamic topology of the network. Connections between units can be created or removed dynamically during the learning phase according to a precise criterion that depends on both the data and the history of the model. This dynamic reconfiguration allows the model to faithfully map the topology of the data as opposed to, for example, a regular self-organising map [176] that uses a fixed topology.

A couple of years before this model was introduced, pruning algorithms [177] were popularized by the Optimal Brain Damage algorithm [178] that aim at finding the optimal size (in terms of the number of connections) of a feed-forward model in order to establish a trade-off between the complexity and the error magnitude of the resulting model. This algorithm starts from a fully connected feed-forward model (multi-layer perceptron) and removes, after training, the weights that have the lowest saliency. With the advent of deep-learning during the past decade and the inflating size of models, there has been a renewed interest in such pruning techniques [179]. However, pruning is the simpler side of structural plasticity since you start from a situation where you can objectify the influence of a neuron A onto a neuron B based on the existence of a connection between them. The careful analysis of this influence can then help to decide if the connection can be removed or not.

On the other hand, the case of sprouting is quite different because there is a need to establish a new connection between a neuron A and a neuron B that are, by definition, not connected. This implies that, from a structural point of view, this selection cannot be based on the correlation of activity, for instance, since no connection exists. Hence, one obvious way to perform the selection is to randomly choose the source and the target. This is the technique used in a number of generative models that search for the best architecture [180]. There exist however non random techniques, such as marker induction, as proposed by [181]. In this model, markers are used to induce presynaptic fibers to connect to the post-synaptic sheet. This results in a neighbouring presynaptic region to connect to a neighbouring postsynaptic region in an ordered fashion and this model is used to explain the formation of ordered retinotectal projections in amphibians and fishes. There exist similar models for the development of the retino-geniculo cortical pathway [182] and more generally, the formation of topographic maps [183, 184]. In addition to several widely applicable advantages of structural plasticity such as optimising energy consumption (as 
discussed in section 4), structural plasticity, in the case of topographic maps (e.g. visual maps), can help to embed pattern of synaptic strengths in the network topology [185] and may explain the existence of a critical period as well. However, from a computational point of view, the advantage of such structural plasticity compared to, for instance, an initially fully connected model that is later pruned, is not clear. Departing from the pure formation of topographical maps, the model by Kappel et al. [186] explains and exploits structural plasticity to give a functional account on the development of a dedicated architecture for solving reward based tasks. As explained by the authors, this model uses reward-driven network plasticity as continuous sampling of network configurations that results in policy sampling. This model is interesting because it provides a functional interpretation of structural plasticity and justifies its presence. Without such plasticity, it would be more difficult or longer for the model to solve the task. However, we suggest that the functional role of the sprouting phase should be considered beyond the formation of topographic maps.

Now while several advantages of structural plasticity have been noted across the literature, ranging from energy efficiency to optimisation of information storage (as discussed in section 4), we investigate if structural plasticity, within a specific circuitry, contributes directly to the function of said circuitry. In order to look into the potential direct functional advantages of structural plasticity, we scrutinize a hypothesis that is gaining popularity in the literature. This hypothesis states that the development of automatized skills, such as song production, is driven by the basal ganglia through dopamine-modulated reinforcement learning in order to guide learning in a parallel cortical pathway, which eventually governs the production of the skill [187, 188, 105].

From a theoretical perspective, this can be considered as a dual learning system, one is reactive and dependent on instantaneous reward (reinforcement learning) while the other is much slower and independent of reward but can strongly bias the output. Reinforcement learning builds an approximation of the local error contour via its exploration techniques, and can implement gradient descent in its pursuit to maximize the expected reward (or minimise the error) [189]. A strong parallel can be drawn between such a system and the notion of momentum in the field of machine learning. Momentum is a simple and popular technique in supervised learning for improving stochastic gradient descent and to escape local minima [190]. This technique averages the last few gradients in order to maintain a consistent direction of the gradient. This has been proven to work better and faster than pure stochastic gradient descent. Very recently, such usage of momentum has been adapted to the framework of reinforcement learning in the case of the value iteration algorithm [191] but the technique can be adapted to any reinforcement learning algorithm, including actor-critic architectures. For this latter case, it is a matter of replacing the critic by the average of successive critics.

Now, we hypothesise a role for structural plasticity in directly aiding the process of song learning, by building an analogy between momentum-guided reinforcement learning and the dual pathway hypothesis for avian vocal learning. As we have seen in earlier sections, in the case of songbirds, the vocal learning circuitry comprises of two parallel pathways. The primary cortical motor pathway (involving the $\mathrm{HVC}$ and RA) is believed to be responsible for controlling song generation, while a secondary BG-thalamo-cortical pathway (involving the LMAN, Area X and DLM) functions as a tutor and sends signals to influence the course of vocal production. Area X receives strong dopaminergic innervation from the midbrain, which provides an online evaluation of song quality [85]. Moreover, there is experimental evidence showing activity-dependent synaptic plasticity at HVC and LMAN projections to spiny neurons in area X [192]. Thus, along with LMAN-driven variability, Area X forms an ideal site to facilitate reinforcement learning. On the other hand, activity dependent synaptic potentiation and depression have also been described at the HVC-RA synapses[193]. This provides an avenue for synaptic plasticity based on Hebbian 

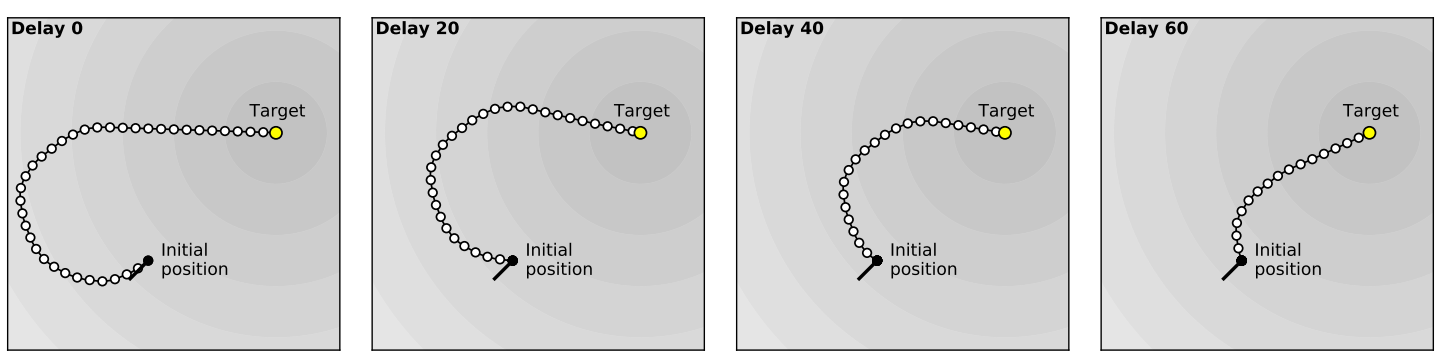

Figure 3: Momentum guided reinforcement learning can be understood using an analogy of velocity and acceleration. In this illustration, velocity corresponds to Hebbian learning, which represents the momentum of the trajectory, while acceleration corresponds to reinforcement learning and can modify the velocity vector up to a certain degree ( $10 \%$ in the figure). The illustration simulates the trajectory from a fixed initial position to a target, when the direction of the velocity is continually modified by the acceleration. In the figure, four trajectories with increasing initial delays (left to right) are shown. These delays correspond to the moment when velocity is activated/allowed to initiate the trajectory. Before that, the acceleration influences the direction of the velocity, however without any displacement. When there's no delay, the initial velocity vector can potentially drive the trajectory away from the target (as shown in this specific example), while with longer delays, the acceleration can explore and identify a more conducive direction in order to exhibit a more direct trajectory to the target.

learning, or co-activation of pre-synaptic and post-synaptic terminals, within the cortical motor pathway. Models such as Teşileanu, Ölveczky, and Balasubramanian [194], also, use STDP-like learning mechanisms, modulated by the parallel BG pathway, to describe the functioning of the cortical pathway. Similar to the mammalian literature discussed above, there is a range of empirical evidence to support the hypothesis that the tutor signals from the BG-thalamo-cortical pathway guide song learning and are eventually consolidated within the cortical pathway[102, 164]. In addition to the synaptic plasticity described in the two parallel pathways, as discussed previously, it has been observed that the HVC axons wait at the dorsal border of RA and enter the RA to form synapses only after the formation of the BG-thalamo-cortical loop [119, 122]. This leads us to investigate if this delayed circuit completion, induced by structural plasticity, might, in fact, have a crucial functional role in the process of vocal learning using the dual pathway architecture.

Drawing an analogy with momentum-guided reinforcement learning, the BG pathway biases the motor output towards the target syllable using reinforcement learning, akin to the role of acceleration in the aforementioned illustration, as both play the role of modulating the direction of the system. The growth of the slower and more stable cortical pathway can be compared to the role of velocity, where both maintain a steady direction in the learning phase. They provide a central locus around which acceleration/the BG loop are able to explore locally and bias the output. The local changes made by acceleration/the BG loop are gradually incorporated and consolidated within velocity/the cortical pathway. Within this analogy, we extend the findings of the illustration to the dual pathway system. We have observed that the slowly-modulated velocity parameter can govern the direction of the system, and can thus provide the momentum required to escape local minima by countering the effect of the acceleration parameter that would preferentially guide the system towards the local minima. This overshooting of shallow local minima in an attempt to reach the global minimum could be a potential role fulfilled by the development of the cortical pathway and the transfer of information from the sub-cortical to cortical pathways. Moreover, in the absence of momentum (as simulated using initiation delays), acceleration can 
exercise comparatively greater influence over velocity, which could, in turn, help find a more conducive direction for the system to begin its trajectory. Thus, during the early babbling stage of vocal learning, where the BG loop is formed, but the cortical pathway isn't yet fully formed, the avian BG can explore the sensorimotor space and find a desirable direction with respect to the local contour. Such early exploration would result in the system moving more rapidly in the chosen direction, eventually, when momentum kicks in or, in this case, when the HVC axons innervate the RA. Considering the cortical motor pathway to implement an equivalent of the notion of momentum, it might be necessary for this pathway to be active only after the initial direction of the gradient is determined. The alternative being a momentum, in a random initial direction, that may cause delays in learning, or ultimately drive the learning away from the actual target. Hence, the developmental delay facilitates an initial period of high exploration, which could be hindered by the presence of a comparatively change-resistant cortical pathway. The delay in the development of cortical pathway, thus, might have a crucial role in controlling the exploration-exploitation trade-off by allowing for an initial predominantly exploratory phase, while additionally assisting in escaping local minima in the later stages of sensorimotor learning. Providing this auxiliary support to gradient descent could be a potential functional role for the otherwise unaccountable delay observed in the innervation of RA neurons by HVC axons.

The comparison of the structural plasticity within the cortical motor pathway with the technique of momentum is also in agreement with a prevalent hypothesis (discussed in section 3) that the innervation of RA by the HVC axons causes an increased inhibitory nature of the RA, which in turn diminishes the influence of the mainly NMDA receptor-based LMAN inputs to the RA [142, 164]. The development of the cortical pathway, thus, slowly diminishes the exploratory LMAN influence and, over time renders the vocal production to be more stereotypical in nature.

While this hypothesis might be difficult to test at the experimental level, it is much more feasible to simulate a comparison between a random initial momentum and a late gradient-directed momentum. Our prediction is that in the former case, learning would be deeply hindered, as illustrated in Figure 3, using an accelerated velocity analogy. It is to be noted that the illustration has been made in 2D and may misleadingly suggest that the initial velocity vector has a fair chance of pointing in the right direction. However, in higher dimensions, this probability would be much lower and therefore, the delay in the activation of the Hebbian-based pathway would be functionally critical ${ }^{2}$.

\section{Conclusion}

Beyond the specific case of birds, for which non-seasonal structural plasticity has been hypothesised to be critical for both acquisition and automatization in juvenile songbirds, we may question to what extent such structural plasticity is similarly critical in vertebrates and more specifically, in mammals. The similarity in brain organization between birds and mammals is indeed striking, especially when considering the cortex-basal ganglia loop. We explained in [196] how the development of automatized skills relies on the BG teaching cortical circuits and is actually a late feature linked with the development of a specialized cortex or pallium that evolved in parallel in different taxa. Alongside this hypothesis, there is a growing number of computational models of decision making that takes into account this dual pathway hypothesis, where the BG acts as a general training machine for cortico-cortical connections [112]. In this context, it is natural to wonder if the initial delay in connection might exist as well in other species and if this would provide

\footnotetext{
${ }^{2}$ The scripts are available on https://github.com/rsankar9/Review-momentum-illustration and archived in a Zenodo repository https://doi.org/10.5281/zenodo.4063714 [195]
} 
a similar benefit in early learning. In humans, this would correspond to the phase preceding the babbling phase that is known to be characterized by rapid structural and functional changes. However, as explained by Vasung et al. [197], the study of early human brain development remains a challenge.

Beyond the interactions between the basal ganglia and the cortex, other learning-related brain circuits function with a 'two-stage' learning process where the initial learning stages rely on subcortical structures while long-term memory is imprinted in neocortical areas through online and/or off-line training of cortical networks by the subcortical regions. Concerning motor learning, BG and cerebellar circuits may teach neocortical motor areas where the motor programs are engrained for long-term storage of motor tasks [198]. Here, we have shown how BG motor output matures earlier than cortico-cortical connections in songbirds, and how this may be beneficial for song learning. Similarly, the cerebello-neocortical circuit driving motor cerebellar-dependent learning may mature before the cortico-cortical connection in motor areas of the motor cortex are mature enough to store the motor memories. Purely speculative, this hypothesis could be tested experimentally by comparing the contribution of cerebellar and neocortical circuits to motor learning across development. Whether delaying the maturation of cortico-cortical connections benefits learning could be tested further in theoretical models of the BG-cortical and cerebello-cortical networks [199].

For declarative memory, the experimentally confirmed 'two-stage' theory [200, 201, 202] posits that new memories are transiently encoded in the hippocampus before they are gradually transferred in the prefrontal cortex for long-term retention. The hippocampus has appeared before the neocortex in evolution, and it is tempting to speculate that ontogeny partially recapitulates evolution with an early maturation of hippocampal circuits involved in declarative memory before the maturation of the prefrontal cortex, which only terminates in adolescence or even later [203]. Such two-stage motor learning and episodic memory formation continues in adulthood, albeit with diminished influence of the cerebullum and hippocampus, respectively. Whether such twostage developmental process could be beneficial for the development of memory circuits, during development, remains to be investigated both experimentally and theoretically.

\section{Acknowledgements}

We thank Dr. Nicolas Giret and Dr. Thomas Boraud for their valuable feedback on the manuscript.

\section{Funding}

This work was partially funded by grant ANR-17-CE24-0036 and ANR-16-CE37-0020-01.

\section{References}

1. A. Citri and R. C. Malenka. "Synaptic plasticity: Multiple forms, functions, and mechanisms." In: Neuropsychopharmacology 33.1 (2008), pp. 18-41.

2. D. Debanne. "Plasticity of neuronal excitability in vivo." In: fournal of Physiology 587.13 (2009), pp. 3057-3058.

3. D. V. Buonomano and M. M. Merzenich. "Cortical plasticity: From synapses to maps." In: Annual Review of Neuroscience 21 (1998), pp. 149-186.

4. M. Butz, F. Wörgötter, and A. van Ooyen. "Activity-dependent structural plasticity.” In: Brain Research Reviews 60.2 (2009), pp. 287-305.

5. P. M. Lledo, M. Alonso, and M. S. Grubb. "Adult neurogenesis and functional plasticity in neuronal circuits." In: Nature Reviews Neuroscience 7.3 (2006), pp. 179-193.

6. C. Portera-Cailliau, R. M. Weimer, V. De Paola, P. Caroni, and K. Svoboda. "Diverse modes of axon elaboration in the developing neocortex." In: PLoS Biology 3.8 (2005). 
7. P. R. Huttenlocher et al. "Synaptic density in human frontal cortex-developmental changes and effects of aging.” In: Brain Res 163.2 (1979), pp. 195-205.

8. P. R. Huttenlocher, C. de Courten, L. J. Garey, and H. Van der Loos. "Synaptogenesis in human visual cortex-evidence for synapse elimination during normal development." In: Neuroscience letters 33.3 (1982), pp. 247 252.

9. P. S. Eriksson, E. Perfilieva, T. Björk-Eriksson, A.-M. Alborn, C. Nordborg, D. A. Peterson, and F. H. Gage. "Neurogenesis in the adult human hippocampus." In: Nature medicine 4.11 (1998), pp. 1313-1317.

10. A. Datwani, T. Iwasato, S. Itohara, and R. S. Erzurumlu. "NMDA Receptor-Dependent Pattern Transfer from Afferents to Postsynaptic Cells and Dendritic Differentiation in the Barrel Cortex." In: Molecular Cell Neuroscience 21.3 (2002), pp. 477-492.

11. R. B. Livingston. "Brain Mechanisms in Conditioning and Learning." In: 4.3 (1966), pp. 235-347.

12. R. H. Wurtz, V. F. Castellucci, and J. M. Nusrala. "Synaptic Plasticity: The Effect of the Action Potential in the Postsynaptic Neuron.” In: Experimental Neurology 18.3 (1967), pp. 350-368.

13. T. V. Bliss and T. Lomo. "Long-lasting potentiation of synaptic transmission in the dentate area of the unanaesthetized rabbit following stimulation of the perforant path." In: The fournal of Physiology 232.2 (1973), pp. 331356.

14. F. W. Kerr. "Structural and functional evidence of plasticity in the central nervous system." In: Experimental Neurology 48.3 PART 2 (1975), pp. 16-31.

15. L. T. Rutledge. "The effects of denervation and stimulation upon synaptic ultrastructure." In: fournal of Comparative Neurology 178.1 (1978), pp. 117-128.

16. A. van Ooyen and E. Butz-Ostendorf Markus. The rewiring brain: a computational approach to structural plasticity in the adult brain. Academic Press, 2017.

17. H. Markram, J. Lübke, M. Frotscher, and B. Sakmann. "Regulation of synaptic efficacy by coincidence of postsynaptic APs and EPSPs.” In: Science 275.5297 (1997), pp. 213-215.

18. G.-q. Bi and M.-m. Poo. "Synaptic modifications in cultured hippocampal neurons: dependence on spike timing, synaptic strength, and postsynaptic cell type." In: Journal of neuroscience 18.24 (1998), pp. 10464-10472.

19. W. Gerstner, R. Kempter, J. L. Van Hemmen, and H. Wagner. "A neuronal learning rule for sub-millisecond temporal coding." In: Nature 383.6595 (1996), pp. 76-78.

20. M. Hartley, N. Taylor, and J. Taylor. "Understanding spike-time-dependent plasticity: A biologically motivated computational model.” In: Neurocomputing 69.16-18 (2006), pp. 2005-2016.

21. H. Z. Shouval, M. F. Bear, and L. N. Cooper. "A unified model of NMDA receptor-dependent bidirectional synaptic plasticity.” In: Proceedings of the National Academy of Sciences 99.16 (2002), pp. 10831-10836.

22. G. M. Innocenti and D. J. Price. "Exuberance in the development of cortical networks." In: Nature Reviews Neuroscience 6.12 (2005), pp. 955-965.

23. Y. Bernardinelli, I. Nikonenko, and D. Muller. "Structural plasticity: Mechanisms and contribution to developmental psychiatric disorders.” In: Frontiers in Neuroanatomy 8.November (2014), pp. 1-9.

24. G. Chechik, I. Meilijson, and E. Ruppin. "Synaptic pruning in development: a computational account." In: Neural computation 10.7 (1998), pp. 1759-1777.

25. D. B. Chklovskii, B. Mel, and K. Svoboda. "Cortical rewiring and information storage." In: Nature 431.7010 (2004), pp. 782-788.

26. A. Knoblauch and F. T. Sommer. "Structural plasticity, effectual connectivity, and memory in cortex." In: Frontiers in neuroanatomy 10 (2016), p. 63.

27. R. Spiess, R. George, M. Cook, and P. U. Diehl. "Structural plasticity denoises responses and improves learning speed.” In: Frontiers in computational neuroscience 10 (2016), p. 93.

28. J. V. Le Bé and H. Markram. "Spontaneous and evoked synaptic rewiring in the neonatal neocortex." In: Proceedings of the National Academy of Sciences of the United States of America 103.35 (2006), pp. 13214-13219.

29. G. Raisman. "Neuronal plasticity in the septal nuclei of the adult rat." In: Brain Research 14.1 (1969), pp. 25-48.

30. J. T. Trachtenberg, B. E. Chen, G. W. Knott, G. Feng, J. R. Sanes, E. Welker, and K. Svoboda. "Long-term in vivo imaging of experience-dependent synaptic plasticity in adult cortex.” In: Nature 420.6917 (2002), pp. 788-794.

31. M. De Roo, P. Klauser, and D. Muller. "LTP promotes a selective long-term stabilization and clustering of dendritic spines." In: PLoS Biology 6.9 (2008), pp. 1850-1860.

32. T. Xu, X. Yu, A. J. Perlik, W. F. Tobin, J. A. Zweig, K. Tennant, T. Jones, and Y. Zuo. "Rapid formation and selective stabilization of synapses for enduring motor memories.” In: Nature 462.7275 (2009), pp. 915-919.

33. T. Van Kerkoerle, S. A. Marik, S. M. Z. A. Borgloh, and C. D. Gilbert. "Axonal plasticity associated with perceptual learning in adult macaque primary visual cortex." In: Proceedings of the National Academy of Sciences of the United States of America 115.41 (2018), pp. 10464-10469.

34. M. J. Geden, S. E. Romero, and M. Deshmukh. "Apoptosis versus Axon Pruning: Molecular Intersection of Two Distinct Pathways for Axon Degeneration.” In: (2019), pp. 3-8.

35. M. Tessier-Lavigne and C. S. Goodman. "The Molecular Biology of Axon Guidance." In: Science 274.5290 (1996), pp. 1123-1133. eprint: https://science. sciencemag.org/content/274/5290/1123. full.pdf. 
36. A. Pascual-Leone and F. Torres. "Plasticity of the sensorimotor cortex representation of the reading finger in braille readers." In: Brain 116.1 (1993), pp. 39-52.

37. C. D. Gilbert. "Adult cortical dynamics." In: Physiological Reviews 78.2 (1998), pp. 467-485.

38. E. Gould. "How widespread is adult neurogenesis in mammals?" In: Nature Reviews Neuroscience 8.6 (2007), pp. 481-488.

39. M. F. Paredes, S. F. Sorrells, J. M. Garcia-Verdugo, and A. Alvarez-Buylla. "Brain size and limits to adult neurogenesis." In: Journal of Comparative Neurology 524.3 (2016), pp. 646-664.

40. C. L. Pytte. "Adult neurogenesis in the songbird: Region-specific contributions of new neurons to behavioral plasticity and stability." In: Brain, Behavior and Evolution 87.3 (2016), pp. 191-204.

41. E. Bufill, J. Agustí, and R. Blesa. "Human neoteny revisited: The case of synaptic plasticity." In: American fournal of Human Biology 23.6 (2011), pp. 729-739.

42. J. R. Boivin, D. J. Piekarski, A. W. Thomas, and L. Wilbrecht. "Adolescent pruning and stabilization of dendritic spines on cortical layer 5 pyramidal neurons do not depend on gonadal hormones." In: Developmental Cognitive Neuroscience 30.January (2018), pp. 100-107.

43. T. K. Hensch. "Critical period plasticity in local cortical circuits." In: Nature Reviews Neuroscience 6.11 (2005), pp. 877-888.

44. T. Nakamori, F. Maekawa, K. Sato, K. Tanaka, and H. Ohki-Hamazaki. "Neural basis of imprinting behavior in chicks.” In: Development, Growth \& Differentiation 55.1 (Jan. 2013), pp. 198-206.

45. M. H. Patton, J. A. Blundon, and S. S. Zakharenko. "Rejuvenation of plasticity in the brain: opening the critical period." In: Current Opinion in Neurobiology 54 (2019), pp. 83-89.

46. D. H. Hubel and T. N. Wiesel. "Brain mechanisms of vision." In: Scientific American 241.3 (1979), pp. 150-162.

47. K. P. Berry and E. Nedivi. "Experience-dependent structural plasticity in the visual system." In: Annual review of vision science 2 (2016), pp. 17-35.

48. Y. Yazaki-Sugiyama. "Neuronal mechanisms regulating the critical period of sensory experience-dependent song learning." In: Neuroscience Research 140 (2019), pp. 53-58.

49. M. Migaud, M. Batailler, D. Pillon, I. Franceschini, and B. Malpaux. "Seasonal changes in cell proliferation in the adult sheep brain and pars tuberalis." In: Journal of Biological Rhythms 26.6 (2011), pp. 486-496.

50. B. Kolb and R. Gibb. Brain Plasticity and Behaviour in the Developing Brain. 2011.

51. M. P. Forrest, E. Parnell, and P. Penzes. "Dendritic structural plasticity and neuropsychiatric disease." In: Nature Reviews Neuroscience 19.4 (2018), pp. 215-234.

52. S. Steven and C. Blakemore. "Cortical Plasticity in the Adult Human Brain." In: The cognitive neurosciences (3rd ed.). (2004), pp. 1243-1254.

53. M. Bosch and Y. Hayashi. "Structural plasticity of dendritic spines." In: Current opinion in neurobiology 22.3 (2012), pp. 383-388.

54. T. L. Lewis Jr, J. Courchet, and F. Polleux. "Cellular and molecular mechanisms underlying axon formation, growth, and branching.” In: Fournal of Cell Biology 202.6 (2013), pp. 837-848.

55. E. Campanac and D. Debanne. "Spike timing-dependent plasticity: a learning rule for dendritic integration in rat CA1 pyramidal neurons." In: The Journal of physiology 586.3 (2008), pp. 779-793.

56. A. Belmeguenai, E. Hosy, F. Bengtsson, C. M. Pedroarena, C. Piochon, E. Teuling, Q. He, G. Ohtsuki, M. T. De Jeu, Y. Elgersma, et al. "Intrinsic plasticity complements long-term potentiation in parallel fiber input gain control in cerebellar Purkinje cells." In: fournal of Neuroscience 30.41 (2010), pp. 13630-13643.

57. P. Sah and J. M. Bekkers. "Apical dendritic location of slow afterhyperpolarization current in hippocampal pyramidal neurons: implications for the integration of long-term potentiation." In: Journal of Neuroscience 16.15 (1996), pp. 4537-4542.

58. D. Saar, Y. Grossman, and E. Barkai. "Reduced after-hyperpolarization in rat piriform cortex pyramidal neurons is associated with increased learning capability during operant conditioning." In: European fournal of Neuroscience 10.4 (1998), pp. 1518-1523.

59. W. C. Oh, T. C. Hill, and K. Zito. "Synapse-specific and size-dependent mechanisms of spine structural plasticity accompanying synaptic weakening." In: Proceedings of the National Academy of Sciences of the United States of America 110.4 (2013).

60. B. Leuner and E. Gould. "Structural plasticity and hippocampal function." In: Annual review of psychology 61 (2010), pp. 111-140.

61. N. Schaefer et al. "The malleable brain: plasticity of neural circuits and behavior - a review from students to students." In: Journal of Neurochemistry 142.6 (2017), pp. 790-811.

62. O. Güntürkün. "The convergent evolution of neural substrates for cognition." In: Psychological Research 76.2 (2012), pp. 212-219.

63. R. Mooney. "Neural mechanisms for learned birdsong." In: Learning and Memory 16.11 (2009), pp. 655-669.

64. K. Tschida and R. Mooney. "The role of auditory feedback in vocal learning and maintenance." In: Current Opinion in Neurobiology 22.2 (2012), pp. 320-327.

65. O. Güntürkün and T. Bugnyar. "Cognition without Cortex." In: Trends in Cognitive Sciences 20.4 (2016), pp. 291303 
66. H. J. Karten. "Vertebrate brains and evolutionary connectomics: On the origins of the mammalian 'neocortex'." In: Philosophical Transactions of the Royal Society B: Biological Sciences 370.1684 (2015).

67. A. J. Doupe and P. K. Kuhl. "BIRDSONG AND HUMAN SPEECH: Common Themes and Mechanisms." In: Annual Review of Neuroscience 22.1 (1999), pp. 567-631.

68. D. F. Sherry and J. S. Hoshooley. "Seasonal hippocampal plasticity in food-storing birds." In: Philosophical Transactions of the Royal Society B: Biological Sciences 365.1542 (2010), pp. 933-943.

69. G. F. Striedter. "Bird brains and tool use: Beyond instrumental conditioning." In: Brain, Behavior and Evolution 82.1 (2013), pp. 55-67.

70. P. H. Boucherie, M. C. Loretto, J. J. Massen, and T. Bugnyar. "What constitutes "social complexity" and "social intelligence" in birds? Lessons from ravens." In: Behavioral Ecology and Sociobiology 73.1 (2019).

71. P. A. Downing, A. S. Griffin, and C. K. Cornwallis. "Group formation and the evolutionary pathway to complex sociality in birds." In: Nature Ecology and Evolution 4.3 (2020), pp. 479-486.

72. I. M. Pepperberg. "Cognitive and communicative abilities of Grey parrots." In: Applied Animal Behaviour Science 100.1-2 (Oct. 2006), pp. 77-86.

73. H. Prior, A. Schwarz, and O. Gunturkun. "Mirror-Induced Behavior in the Magpie (Pica pica): Evidence of Self-Recognition.” In: Plos One 6.8 (2008), pp. 1642-1650.

74. O. Güntürkün. "The avian 'prefrontal cortex' and cognition." In: Current Opinion in Neurobiology 15.6 (2005), pp. 686-693.

75. C. Herold, P. Schlömer, I. Mafoppa-Fomat, J. Mehlhorn, K. Amunts, and M. Axer. "The hippocampus of birds in a view of evolutionary connectomics.” In: Cortex 118 (2019), pp. 165-187.

76. A. Reiner, L. Medina, and C. L. Veenman. "Structural and functional evolution of the basal ganglia in vertebrates.” In: Brain Research Reviews 28.3 (1998), pp. 235-285.

77. A. Reiner et al. "Revised Nomenclature for Avian Telencephalon and Some Related Brainstem Nuclei." In: Journal of Comparative Neurology 473.3 (2004), pp. 377-414. arXiv: NIHMS150003.

78. L. Medina and A. Reiner. "Do birds possess homologues of mammalian primary visual, somatosensory and motor cortices?” In: Evolution of Nervous Systems 23 (2000), pp. 1-12.

79. Y. Wang, A. Brzozowska-Prechtl, and H. J. Karten. "Laminar and columnar auditory cortex in avian brain." In: Proceedings of the National Academy of Sciences of the United States of America 107.28 (2010), pp. 12676-12681.

80. A. Calabrese and S. M. Woolley. "Coding principles of the canonical cortical microcircuit in the avian brain." In: Proceedings of the National Academy of Sciences of the United States of America 112.11 (2015), pp. 3517-3522.

81. A. B. Butler, A. Reiner, and H. J. Karten. "Evolution of the amniote pallium and the origins of mammalian neocortex." In: Annals of the New York Academy of Sciences 1225.1 (2011), pp. 14-27.

82. J. F. Montiel, N. A. Vasistha, F. Garcia-Moreno, and Z. Molnár. "From sauropsids to mammals and back: New approaches to comparative cortical development." In: Journal of Comparative Neurology 524.3 (2016), pp. 630645.

83. A. J. Doupe, D. J. Perkel, A. Reiner, and E. A. Stern. "Birdbrains could teach basal ganglia research a new song." In: Trends in Neurosciences 28.7 (2005), pp. 353-363.

84. S. W. Bottjer and F. Johnson. "Circuits, hormones, and learning: Vocal behavior in songbirds." In: fournal of Neurobiology 33.5 (1997), pp. 602-618.

85. D. A. Lewis and S. R. Sesack. "Dopamine systems in the primate brain." In: Handbook of Chemical Neuroanatomy 13 (1997), pp. 263-375.

86. M. A. Farries and D. J. Perkel. "A Telencephalic Nucleus Essential for Song Learning Contains Neurons with Physiological Characteristics of Both Striatum and Globus Pallidus.” In: Journal of Neuroscience 22.9 (2002), pp. 3776-3787.

87. G. D. Carrillo and A. J. Doupe. "Is the Songbird Area X Striatal, Pallidal, or Both? An Anatomical Study." In: Journal of Comparative Neurology 473.3 (2004), pp. 415-437.

88. M. Luo and D. J. Perkel. "A GABAergic, strongly inhibitory projection to a thalamic nucleus in the zebra finch song system.” In: Journal of Neuroscience 19.15 (1999), pp. 6700-6711.

89. A. Leblois, Á. L. Bodor, A. L. Person, and D. J. Perkel. "Millisecond timescale disinhibition mediates fast information transmission through an avian basal ganglia loop." In: fournal of Neuroscience 29.49 (2009), pp. 1542015433.

90. K. D. MacDermot et al. "Identification of FOXP2 truncation as a novel cause of developmental speech and language deficits." In: American fournal of Human Genetics 76.6 (2005), pp. 1074-1080.

91. S. Haesler, K. Wada, A. Nshdejan, E. E. Morrisey, T. Lints, E. D. Jarvis, and C. Scharff. "FoxP2 Expression in Avian Vocal Learners and Non-Learners." In: fournal of Neuroscience 24.13 (2004), pp. 3164-3175.

92. M. S. Brainard and A. J. Doupe. "What songbirds teach us about learning." In: Nature 417 (2002), pp. 351-417.

93. R. H. Hahnloser and A. Kotowicz. "Auditory representations and memory in birdsong learning." In: Current Opinion in Neurobiology 20.3 (2010), pp. 332-339.

94. R. H. Hahnloser, A. A. Kozhevnikov, and M. S. Fee. An ultra-sparse code underlies the generation of neural sequences in a songbird. 2002 
95. M. A. Long and M. S. Fee. "Using temperature to analyse temporal dynamics in the songbird motor pathway." In: Nature 456.7219 (2008), pp. 189-194.

96. M. F. Schmidt, J. Mclean, and F. Goller. "Breathing and vocal control: The respiratory system as both a driver and a target of telencephalic vocal motor circuits in songbirds." In: Experimental Physiology 97.4 (2012), pp. 455461.

97. M. S. Brainard and A. J. Doupe. "Translating Birdsong: Songbirds as a Model for Basic and Applied Medical Research.” In: Annual Review of Neuroscience 36.1 (2013), pp. 489-517.

98. G. E. Vates and F. Nottebohm. "Feedback circuitry within a song-learning pathway." In: Neurobiology 92.May (1995), pp. 5139-5143.

99. M. Luo, L. Ding, and D. J. Perkel. "An avian basal ganglia pathway essential for vocal learning forms a closed topographic loop.” In: Journal of Neuroscience 21.17 (2001), pp. 6836-6845.

100. C. Scharff and F. Nottebohm. "A comparative study of the behavioral deficits following lesions of various parts of the zebra finch song system: Implications for vocal learning." In: fournal of Neuroscience 11.9 (1991), pp. 2896-2913.

101. S. W. Bottjer, E. A. Miesner, and A. P. Arnold. "Forebrain Lesions Disrupt Development But Not Maintenance of Song in Passerine Birds.” In: Science 224.4651 (1984), pp. 901-903.

102. B. P. Ölveczky, A. S. Andalman, and M. S. Fee. "Vocal experimentation in the juvenile songbird requires a basal ganglia circuit.” In: PLoS Biology 3.5 (2005), pp. 0902-0909.

103. T. L. Warren, E. C. Tumer, J. D. Charlesworth, and M. S. Brainard. "Mechanisms and time course of vocal learning and consolidation in the adult songbird." In: fournal of Neurophysiology 106.4 (2011), pp. 1806-1821.

104. A. Leblois, B. J. Wendel, and D. J. Perkel. "Striatal dopamine modulates basal ganglia output and regulates social context-dependent behavioral variability through D1 receptors." In: fournal of Neuroscience 30.16 (2010), pp. $5730-5743$.

105. A. S. Andalman and M. S. Fee. "A basal ganglia-forebrain circuit in the songbird biases motor output to avoid vocal errors." In: Proceedings of the National Academy of Sciences of the United States of America 106.30 (2009), pp. 12518-12523.

106. V. Gadagkar and J. H. Goldberg. "Dopamine Neurons Encode Performance Error in Singing Birds.” In: Science 176.1 (2016), pp. 139-148.

107. W. Schultz, P. Dayan, and P. R. Montague. "A neural substrate of prediction and reward." In: Science 275.5306 (1997), pp. 1593-1599.

108. E. Hisey, M. G. Kearney, and R. Mooney. "A common neural circuit mechanism for internally guided and externally reinforced forms of motor learning.” In: Nature Neuroscience 21.4 (2018), pp. 589-597.

109. L. Xiao, G. Chattree, F. G. Oscos, M. Cao, M. J. Wanat, and T. F. Roberts. "A Basal Ganglia Circuit Sufficient to Guide Birdsong Learning." In: Neuron 98.1 (2018), 208-221.e5.

110. L. Ding and D. J. Perkel. "Long-Term Potentiation in an Avian Basal Ganglia Nucleus Essential for Vocal Learning." In: Journal of Neuroscience 24.2 (2004), pp. 488-494.

111. J. N. Reynolds and J. R. Wickens. "A state-dependent trigger for electrophysiological recording at predetermined membrane potentials." In: Journal of Neuroscience Methods 131.1-2 (2003), pp. 111-119.

112. S. Hélie, S. W. Ell, and F. G. Ashby. "Learning robust cortico-cortical associations with the basal ganglia: An integrative review." In: Cortex 64 (2015), pp. 123-135.

113. M. S. Fee and C. Scharff. "The songbird as a model for the generation and learning of complex sequential behaviors.” In: ILAR fournal 51.4 (2010), pp. 362-377.

114. K. Wada et al. "A molecular neuroethological approach for identifying and characterizing a cascade of behaviorally regulated genes." In: Proceedings of the National Academy of Sciences of the United States of America 103.41 (2006), pp. 15212-15217.

115. M. Konishi. "Birdsong: From Behavior to Neuron." In: Annual Review of Neuroscience 8.1 (1985), pp. 125-170.

116. S. W. Bottjer, E. A. Miesner, and A. P. Arnold. "Changes in neuronal number, density and size account for increases in volume of song-control nuclei during song development in zebra finches." In: Neuroscience Letters 67.3 (1986), pp. 263-268.

117. K. Herrmann and H.-J. .-J. Bischof. "Delayed development of song control nuclei in the zebra finch is related to behavioral development." In: Journal of Comparative Neurology 245.2 (1986), pp. 167-175.

118. K. W. Nordeen and E. J. Nordeen. "Projection neurons within a vocal motor pathway are born during song learning in zebra finches." In: Nature 334.6178 (1988), pp. 149-151.

119. K. Herrmann and A. P. Arnold. "The development of afferent projections to the robust archistriatal nucleus in male zebra finches: A quantitative electron microscopic study." In: fournal of Neuroscience 11.7 (1991), pp. 2063-2074.

120. F. Johnson and S. W. Bottjer. "Growth and regression of thalamic efferents in the song-control system of male zebra finches." In: Journal of Comparative Neurology 326.3 (1992), pp. 442-450.

121. F. Sohrabji, E. J. Nordeen, and K. W. Nordeen. "Characterization of neurons born and incorporated into a vocal control nucleus during avian song learning." In: Brain Research 620.2 (1993), pp. 335-338. 
122. R. Mooney and M. Rao. "Waiting periods versus early innervation: The development of axonal connections in the zebra finch song system." In: Journal of Neuroscience 14.11 I (1994), pp. 6532-6543.

123. G. Teuchert-Noodt, K.-H. Breuker, and R. Dawirs. "Neuronal lysosome accumulation in degrading synapses of sensory-motor and limbic subsystems in the duck Anas platyrhynchos: indication of rearrangements during avian brain development?” In: Developmental neuroscience 13.3 (1991), pp. 151-163.

124. D. F. Sherry and S. A. MacDougall-Shackleton. "Seasonal change in the avian hippocampus." In: Frontiers in Neuroendocrinology 37 (Apr. 2015), pp. 158-167.

125. L. D. LaDage, T. C. Roth, and V. V. Pravosudov. "Hippocampal neurogenesis is associated with migratory behaviour in adult but not juvenile sparrows (Zonotrichia leucophrys ssp.)” In: Proceedings of the Royal Society B: Biological Sciences 278.1702 (2011), pp. 138-143.

126. E. A. Brenowitz. "Plasticity of the adult avian song control system." In: Annals of the New York Academy of Sciences 1016 (2004), pp. 560-585.

127. M. M. Mariette and K. L. Buchanan. "Prenatal acoustic communication programs offspring for higher posthatching temperatures in a songbird.” In: Behavioral Ecology 353.6301 (2016), pp. 812-814.

128. N. Amin, A. Doupe, and F. E. Theunissen. "Development of selectivity for natural sounds in the songbird auditory forebrain.” In: Journal of Neurophysiology 97.5 (2007), pp. 3517-3531.

129. A. N. Chen and C. D. Meliza. "Experience-and sex-dependent intrinsic plasticity in the zebra finch auditory cortex during song memorization.” In: Journal of Neuroscience 40.10 (2020), pp. 2047-2055.

130. M. T. Ross, D. Flores, R. Bertram, F. Johnson, and R. L. Hyson. "Neuronal intrinsic physiology changes during development of a learned behavior." In: eneuro 4.5 (2017).

131. S. S. Shank and D. Margoliash. "Sleep and sensorimotor integration during early vocal learning in a songbird." In: Nature 458.7234 (2009), pp. 73-77.

132. K. A. Tschida and R. Mooney. "Deafening drives cell-type-specific changes to dendritic spines in a sensorimotor nucleus important to learned vocalizations." In: Neuron 73.5 (2012), pp. 1028-1039.

133. A. Daou and D. Margoliash. "Intrinsic neuronal properties represent song and error in zebra finch vocal learning." In: Nature communications 11.1 (2020), pp. 1-17.

134. A. Daou and D. Margoliash. "Intrinsic plasticity and birdsong learning." In: Neurobiology of Learning and Memory 180 (2021), p. 107407.

135. I. R. Fiete, W. Senn, C. Z. Wang, and R. H. Hahnloser. "Spike-Time-Dependent Plasticity and Heterosynaptic Competition Organize Networks to Produce Long Scale-Free Sequences of Neural Activity." In: Neuron 65.4 (2010), pp. 563-576.

136. T. F. Roberts, K. A. Tschida, M. E. Klein, and R. Mooney. "Rapid spine stabilization and synaptic enhancement at the onset of behavioural learning." In: Physiology \& behavior 176.1 (2016), pp. 139-148.

137. G. Cornez, E. Jonckers, S. M. ter Haar, A. V. der Linden, C. A. Cornil, and J. Balthazart. "Timing of perineuronal net development in the zebra finch song control system correlates with developmental song learning." In: Proceedings of the Royal Society B: Biological Sciences 285.1883 (July 2018), p. 20180849.

138. S. Iyengar, S. S. Viswanathan, and S. W. Bottjer. "Development of topography within song control circuitry of zebra finches during the sensitive period for song learning." In: Journal of Neuroscience 19.14 (1999), pp. 60376057.

139. A. Alvarez-Buylla, M. Theelen, and F. Nottebohm. "Birth of projection neurons in the higher vocal center of the canary forebrain before, during, and after song learning." In: Proceedings of the National Academy of Sciences of the United States of America 85.22 (1988), pp. 8722-8726.

140. S. W. Bottjer, S. L. Glaessner, and A. P. Arnold. "Ontogeny of brain nuclei controlling song learning and behavior in zebra finches.” In: Journal of Neuroscience 5.6 (1985), pp. 1556-1562.

141. D. Aronov, A. S. Andalman, and M. S. Fee. "A specialized forebrain circuit for vocal babbling in the juvenile songbird.” In: Science 320.5876 (2008), pp. 630-634.

142. J. Garst-Orozco, B. Babadi, and B. P. Ölveczky. "A neural circuit mechanism for regulating vocal variability during song learning in zebra finches.” In: eLife 3 (2014), e03697.

143. L. F. Jacobs. "The economy of winter: Phenotypic plasticity in behavior and brain structure." In: Biological Bulletin 191.1 (1996), pp. 92-100.

144. G. F. Ball and J. Balthazart. "Seasonal and hormonal modulation of neurotransmitter systems in the song control circuit." In: Journal of Chemical Neuroanatomy 39.2 (2010), pp. 82-95.

145. F. Nottebohm, M. E. Nottebohm, L. A. Crane, and J. C. Wingfield. "Seasonal changes in gonadal hormone levels of adult male canaries and their relation to song." In: Behavioral and Neural Biology 47.2 (1987), pp. 197-211.

146. B. A. Alward, J. Balthazart, and G. F. Ball. "Differential effects of global versus local testosterone on singing behavior and its underlying neural substrate." In: Proceedings of the National Academy of Sciences of the United States of America 110.48 (2013), pp. 19573-19578.

147. F. Nottebohm. "A Brain for All Seasons: Cyclical Anatomical Changes in Song Control Nuclei of the Canary Brain.” In: Science 214.4527 (1981), pp. 1368-1370.

148. E. A. Brenowitz. "Comparative approaches to the avian song system." In: Journal of Neurobiology · 4695.December 1997 (1997). 
149. F. Nottebohm, M. E. Nottebohm, and L. Crane. "Developmental and seasonal changes in canary song and their relation to changes in the anatomy of song-control nuclei." In: Behavioral and Neural Biology 46.3 (1986), pp. 445-471.

150. M. L. Caras, M. O’Brien, E. A. Brenowitz, and E. W. Rubel. "Estradiol selectively enhances auditory function in avian forebrain neurons." In: fournal of Neuroscience 32.49 (2012), pp. 17597-17611.

151. G. De Groof, M. Verhoye, V. Van Meir, J. Balthazart, and A. Van Der Linden. "Seasonal rewiring of the songbird brain: An in vivo MRI study." In: European fournal of Neuroscience 28.12 (2008), pp. 2475-2485.

152. C. Del Negro and J. M. Edeline. "Sex and season influence the proportion of thin spike cells in the canary HVc." In: NeuroReport 13.16 (2002), pp. 2005-2009.

153. J. Meitzen, D. J. Perkel, and E. A. Brenowitz. "Seasonal changes in intrinsic electrophysiological activity of song control neurons in wild song sparrows." In: Journal of Comparative Physiology A: Neuroethology, Sensory, Neural, and Behavioral Physiology 193.6 (2007), pp. 677-683.

154. A. D. Tramontin and E. A. Brenowitz. "Seasonal plasticity in the adult brain." In: Trends in Neurosciences 23.6 (2000), pp. 251-258.

155. S. A. Goldman and F. Nottebohm. "Neuronal production, migration, and differentiation in a vocal control nucleus of the adult female canary brain." In: Proceedings of the National Academy of Sciences of the United States of America 80.8 I (1983), pp. 2390-2394.

156. A. Alvarez-Buylla and J. R. Kirn. "Birth, migration, incorporation, and death of vocal control neurons in adult songbirds." In: Journal of Neurobiology 33.5 (1997), pp. 585-601.

157. K. M. Hill and T. J. DeVoogd. "Altered daylength affects dendritic structure in a song-related brain region in red-winged blackbirds.” In: Behavioral and Neural Biology 56.3 (1991), pp. 240-250.

158. J. A. Thompson and F. Johnson. "HVC Microlesions Do Not Destabilize the Vocal Patterns of Adult Male Zebra Finches with Prior Ablation of LMAN.” In: Developmental Neurobiology 67: (2007), pp. 205-218.

159. S. Korsia and S. W. Bottjer. "Chronic testosterone treatment impairs vocal learning in male zebra finches during a restricted period of development." In: Journal of Neuroscience 11.8 (1991), pp. 2362-2371.

160. C. S. Whaling, D. A. Nelson, and P. Marler. "Testosterone-induced shortening of the storage phase of song development in birds interferes with vocal learning." In: Developmental Psychobiology 28.7 (1995), pp. 367376.

161. S. R. y. Cajal. Degeneration \& regeneration of the nervous system. Oxford University Press, 1928.

162. E. C. Tumer and M. S. Brainard. "Performance variability enables adaptive plasticity of 'crystallized' adult birdsong." In: Nature 450.7173 (2007), pp. 1240-1244.

163. J. Alliende, N. Giret, L. Pidoux, C. Del Negro, and A. Leblois. "Seasonal plasticity of song behavior relies on motor and syntactic variability induced by a basal ganglia-forebrain circuit." In: Neuroscience 359 (2017), pp. 49-68.

164. B. P. Ölveczky, T. M. Otchy, J. H. Goldberg, D. Aronov, and M. S. Fee. "Changes in the neural control of a complex motor sequence during learning." In: Journal of Neurophysiology 106.1 (July 2011), pp. 386-397.

165. R. Mooney and M. Konishi. "Two distinct inputs to an avian song nucleus activate different glutamate receptor subtypes on individual neurons." In: Proceedings of the National Academy of Sciences of the United States of America 88.10 (1991), pp. 4075-4079.

166. M. Butz and A. van Ooyen. "A simple rule for dendritic spine and axonal bouton formation can account for cortical reorganization after focal retinal lesions.” In: PLoS computational biology 9.10 (2013), e1003259.

167. M. Butz, I. D. Steenbuck, and A. van Ooyen. "Homeostatic structural plasticity increases the efficiency of small-world networks." In: Frontiers in synaptic neuroscience 6 (2014), p. 7.

168. A. Knoblauch, E. Körner, U. Körner, and F. T. Sommer. "Structural synaptic plasticity has high memory capacity and can explain graded amnesia, catastrophic forgetting, and the spacing effect." In: PloS one 9.5 (2014), e96485.

169. P. Poirazi and B. W. Mel. "Impact of active dendrites and structural plasticity on the memory capacity of neural tissue.” In: Neuron 29.3 (2001), pp. 779-796.

170. A. Stepanyants, P. R. Hof, and D. B. Chklovskii. "Geometry and structural plasticity of synaptic connectivity." In: Neuron 34.2 (2002), pp. 275-288.

171. K. A. Sailor, M. T. Valley, M. T. Wiechert, H. Riecke, G. J. Sun, W. Adams, J. C. Dennis, S. Sharafi, G.-1. Ming, H. Song, et al. "Persistent structural plasticity optimizes sensory information processing in the olfactory bulb." In: Neuron 91.2 (2016), pp. 384-396.

172. T. Lightheart, S. Grainger, and T.-F. Lu. "Spike-timing-dependent construction." In: Neural computation 25.10 (2013), pp. 2611-2645.

173. A. Knoblauch, G. Palm, and F. T. Sommer. "Memory capacities for synaptic and structural plasticity." In: Neural Computation 22.2 (2010), pp. 289-341.

174. A. Knoblauch. "Impact of structural plasticity on memory formation and decline." In: The Rewiring Brain. Elsevier, 2017, pp. 361-386.

175. B. Fritzke. "A Growing Neural Gas Network Learns Topologies." In: Advances in Neural Information Processing Systems 7. Ed. by G. Tesauro, D. S. Touretzky, and T. K. Leen. MIT Press, 1995, pp. 625-632. 
176. T. Kohonen. "Self-organized formation of topologically correct feature maps." In: Biological Cybernetics 43.1 (1982), pp. 59-69.

177. R. Reed. "Pruning algorithms-a survey." In: IEEE Transactions on Neural Networks 4.5 (1993), pp. 740-747.

178. Y. LeCun, J. S. Denker, and S. A. Solla. "Optimal Brain Damage." In: Advances in Neural Information Processing Systems 2. Ed. by D. S. Touretzky. Morgan-Kaufmann, 1990, pp. 598-605.

179. D. Blalock, J. J. G. Ortiz, J. Frankle, and J. Guttag. What is the State of Neural Network Pruning? 2020. arXiv: 2003.03033 [CS.LG].

180. P. J. Angeline, G. M. Saunders, and J. B. Pollack. "An evolutionary algorithm that constructs recurrent neural networks." In: IEEE Transactions on Neural Networks 5.1 (1994), pp. 54-65.

181. D. J. Willshaw and C. V. D. Malsburg. "A marker induction mechanism for the establishment of ordered neural mappings: its application to the retinotectal problem.” In: Philosophical Transactions of the Royal Society of London. B, Biological Sciences 287.1021 (Nov. 1979), pp. 203-243.

182. T. Elliott and N. R. Shadbolt. "A Neurotrophic Model of the Development of the Retinogeniculocortical Pathway Induced by Spontaneous Retinal Waves.” In: The fournal of Neuroscience 19.18 (Sept. 1999), pp. 7951-7970.

183. J. Sirosh and R. Miikkulainen. "Self-organization and functional role of lateral connections and multisize receptive fields in the primary visual cortex." In: Neural Processing Letters 3.1 (Apr. 1996), pp. 39-48.

184. P. A. Bogdan, A. G. D. Rowley, O. Rhodes, and S. B. Furber. "Structural Plasticity on the SpiNNaker Many-Core Neuromorphic System.” In: Frontiers in Neuroscience 12 (July 2018).

185. S. A. Bamford, A. F. Murray, and D. J. Willshaw. "Synaptic rewiring for topographic mapping and receptive field development.” In: Neural Networks 23.4 (May 2010), pp. 517-527.

186. D. Kappel, R. Legenstein, S. Habenschuss, M. Hsieh, and W. Maass. "A Dynamic Connectome Supports the Emergence of Stable Computational Function of Neural Circuits through Reward-Based Learning." In: eneuro 5.2 (Mar. 2018), ENEURO.0301-17.2018.

187. F. G. Ashby, B. O. Turner, and J. C. Horvitz. "Cortical and basal ganglia contributions to habit learning and automaticity." In: Trends in cognitive sciences 14.5 (2010), pp. 208-215.

188. A. Pasupathy and E. K. Miller. "Different time courses of learning-related activity in the prefrontal cortex and striatum.” In: Nature 433.7028 (2005), pp. 873-876.

189. R. S. Sutton, A. G. Barto, et al. Introduction to reinforcement learning. Vol. 135. MIT press Cambridge, 1998.

190. G. Goh. "Why Momentum Really Works." In: Distill (2017).

191. N. Vieillard, B. Scherrer, O. Pietquin, and M. Geist. "Momentum in reinforcement learning." In: International Conference on Artificial Intelligence and Statistics. PMLR. 2020, pp. 2529-2538.

192. L. Ding and D. J. Perkel. "Long-term potentiation in an avian basal ganglia nucleus essential for vocal learning." In: Journal of Neuroscience 24.2 (2004), pp. 488-494.

193. W. H. Mehaffey and A. J. Doupe. "Naturalistic stimulation drives opposing heterosynaptic plasticity at two inputs to songbird cortex." In: Nature neuroscience 18.9 (2015), pp. 1272-1280.

194. T. Teşileanu, B. Ölveczky, and V. Balasubramanian. "Rules and mechanisms for efficient two-stage learning in neural circuits." In: Elife 6 (2017), e20944.

195. R. Sankar, N. P. Rougier, and A. Leblois. rsankar9/Review-momentum-illustration. Version v1.0.0. Mar. 2021.

196. T. Boraud, A. Leblois, and N. P. Rougier. "A natural history of skills." In: Progress in Neurobiology 171 (Dec. 2018), pp. 114-124.

197. L. Vasung, E. A. Turk, S. L. Ferradal, J. Sutin, J. N. Stout, B. Ahtam, P.-Y. Lin, and P. E. Grant. "Exploring early human brain development with structural and physiological neuroimaging." In: NeuroImage 187 (Feb. 2019), pp. 226-254.

198. D. Caligiore et al. "Consensus Paper: Towards a Systems-Level View of Cerebellar Function: the Interplay Between Cerebellum, Basal Ganglia, and Cortex.” In: The Cerebellum 16.1 (Feb. 2016), pp. 203-229.

199. K. Doya. "Complementary roles of basal ganglia and cerebellum in learning and motor control." In: Current Opinion in Neurobiology 10.6 (Dec. 2000), pp. 732-739.

200. D. Marr. "Simple memory: a theory for archicortex." In: Philosophical Transactions of the Royal Society of London. B, Biological Sciences 262.841 (July 1971), pp. 23-81.

201. B. Bontempi, C. Laurent-Demir, C. Destrade, and R. Jaffard. "Time-dependent reorganization of brain circuitry underlying long-term memory storage.” In: Nature 400.6745 (Aug. 1999), pp. 671-675.

202. T. Maviel. "Sites of Neocortical Reorganization Critical for Remote Spatial Memory." In: Science 305.5680 (July 2004), pp. 96-99.

203. K. Teffer and K. Semendeferi. "Human prefrontal cortex: evolution, development, and pathology." In: Progress in brain research. Vol. 195. Elsevier, 2012, pp. 191-218. 